\title{
Recent Advances in Indoor Localization: A Survey on Theoretical Approaches and Applications
}

\author{
Ali Yassin ${ }^{\diamond}$, Youssef Nasser ${ }^{\diamond}$, Mariette Awad ${ }^{\diamond}$, Ahmed Al-Dubai ${ }^{++}$, Ran Liu *, Chau Yuen *, Ronald Raulefs ${ }^{+}$, Elias \\ Aboutanios $^{\S}$ \\ ${ }^{\diamond}$ ECE Department, Faculty of Engineering and Architecture, American University of Beirut, Lebanon \\ ++ School of Computing, Edinburgh Napier University, UK \\ * Engineering Product Development, Singapore University of Technology and Design, Singapore \\ + German AeroSpace Center (DLR), Oberpfaffenhofen, Germany \\ §School of Electrical Engineering and Telecommunications, University of New South Wales, Australia \\ Email:ahy04@aub.edu.lb,youssef.nasser@aub.edu.lb
}

\begin{abstract}
The availability of location information has become a key factor in today's communications systems allowing location based services. In outdoor scenarios, the mobile terminal position is obtained with high accuracy thanks to the Global Positioning System (GPS) or to the standalone cellular systems. However, the main problem of GPS and cellular systems resides in the indoor environment and in scenarios with deep shadowing effects where the satellite or cellular signals are broken. In this paper, we survey different technologies and methodologies for indoor and outdoor localization with an emphasis on indoor methodologies and concepts. Additionally, we discuss in this review different localization-based applications, where the location information is critical to estimate. Finally, a comprehensive discussion of the challenges in terms of accuracy, cost, complexity, security, scalability, etc. is given. The aim of this survey is to provide a comprehensive overview of existing efforts as well as auspicious and anticipated dimensions for future work in indoor localization techniques and applications.

Index Terms-localization, cooperative localization, hybrid data fusion, SLAM, cooperative navigation, fundamental limits, signals of opportunity, ultra-wideband (UWB), game theory, coalition games, wireless sensor network (WSN) and location-based services (LBS).
\end{abstract}

\section{INTRODUCTION}

Modern communications systems aim at providing high data rates with ubiquitous service coverage. Nowadays, the availability of the Mobile Terminal or Unlocated Device (UD) location information at the base stations, i.e. its knowledge by the operators, has become a key factor in enabling communications systems to provide new location based services [1].

Practical localization techniques are based on Time of Arrival (ToA), Time Difference of Arrival (TDoA), Received Signal Strength (RSS) and Angle of Arrival (AoA). In outdoor scenarios, the UD position can be obtained with high accuracy from Global Navigation Satellite Systems (GNSS), such as the Global Positioning System (GPS), or from the standalone cellular systems. However, these positioning systems are severely degraded or may fail altogether in indoor environments where the satellite or cellular signals are interrupted, and in scenarios with deep shadowing effects [2]. Various approaches and methodologies have been proposed to deal with these problems. Hybrid positioning is a well-known approach for positioning that exhibits sufficient accuracy and coverage [3]. In this respect, combined localization approaches are extensively proposed in the literature to solve the hearability problem in indoor scenarios.

Another potential candidate for critical scenarios consists of the class of heterogeneous approaches that combine different radio access technologies (such as cellular systems like $3 \mathrm{G}$ and $4 \mathrm{G}$, WLAN, and WiMAX), as shown in Figure 1. Indeed, techniques based on combinations of cellular and WLAN networks have recently received increasing interests from both the localization and communication communities [4][5]. This is not only due to the request made by Federal Communication Commission (FCC) regarding the accurate localization of the UDs, but also because of the many applications that are location sensitive such as billing, fleet management, and mobile yellow pages [6].

Although any positioning techniques could be exploited in indoor scenarios and homogeneous networks, there are practical limits on the combination of these techniques as well as on the minimal number of anchor nodes (AN) that can be used in such scenarios [7]. For instance, in many cases, only one or two ANs are able to communicate with the UD. Hence, new techniques based on hybrid data fusion and/or heterogeneous access are proposed and analyzed in this case.

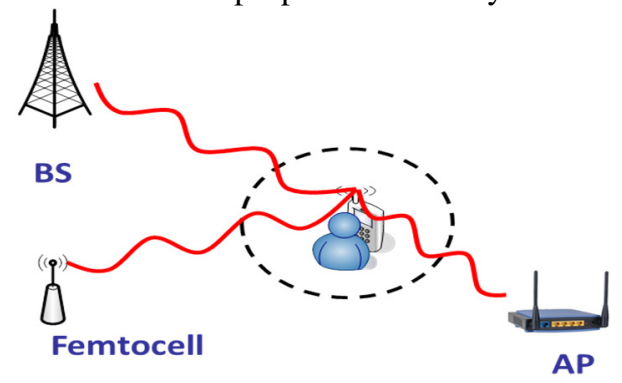

Figure 1. Heterogeneous Scenario (AP: stands for access point, BS stands for Base Station)

In this paper, we provide a review on recent techniques and concepts used to improve localization with their fundamental limits, challenges and applications with a particular focus on 
indoor environments. Although reviews on localization techniques are available in the literature [8]-[15], these are either narrow in focus or have been overtaken by significant technological advances. Thus, the survey in [12] is somewhat outdated, whereas the authors of [8] focus only on ultrasonic positioning systems. The work in [10] describes relatively recent localization techniques but does not explore the future trends, challenges, and applications. The works of [13] and [14] review various technologies, such as WLAN, used for indoor positioning in addition to different positioning technique with the metrics used to assess the performance, such as the estimation accuracy of positioning. However, they do not discuss positioning neither from the perspective of energy efficiency nor from the perspective of a requirement in recent applications, such as ambient assisted and health living applications. Additionally, they do not explore advanced methodologies used to enhance localization, such as cooperative localization and data fusion techniques. The survey in [15] provides remarkable classification of different fingerprint-based outdoor localization approaches, discussing how each method works. So, we aim to present a survey that is restricted neither for fingerprinting-based techniques nor for outdoor localization. As well, the rapid evolution of methodologies and technologies in this domain and the need for a comprehensive and up-to-date survey of the approaches, applications and future trends, provide the motivation for this review paper.

To summarize, a number of aspects differentiate this paper from existing works; first, we review advanced localization techniques and positioning systems for indoor and outdoor environments. Second, we discuss recent methodologies such as data fusion and cooperative techniques used to enhance the accuracy of localization. Third, we present an overview of machine learning techniques that have recently been adopted for localization purposes. Fourth, we describe various localization-based applications from different fields. Finally, we present a comprehensive list of localization challenges foreseen in the future mainly in next generation $5 \mathrm{G}$ networks. The rest of the paper is organized as follows. In section II ,we discuss the fundamental limits of localization in indoor environments and describe the basic localization techniques in section III. Also, we discuss the state-of-the-art system-based localization techniques with the challenges in terms of energy consumption and positioning accuracy in section IV. Then, we review cooperative localization and hybrid data fusion techniques in section V. In section VI we turn our attention to the use of game theory generally and coalition games for localization. In section VII we explore various localizationbased applications. We present in section VIII a comparative study on the accuracy, range and techniques used for different localization systems. Finally, perspectives and challenges of recent advancements in indoor localization are discussed in section IX.

\section{FUNDAMENTAL LIMITS OF LOCALIZATION IN INDOOR ENVIRONMENTS}

Position information is usually provided by global navigation satellite systems, such as GPS or the European satellite navigation system Galileo. However, the accuracy of positioning is affected by the environment, especially in indoor scenarios or dense urban areas where localization using GNSS can be inaccurate or even impossible due to the interruption of the connection with the required satellites. Hence, alternative localization algorithms have to be used to estimate UD position with high accuracy.

This has been primarily achieved using radio signals offered by terrestrial radio access networks. Typically, these positioning strategies comprise a two-stage positioning system consisting of a ranging phase where nodes use distance dependent signal relation, such as RSS, ToA, AoA, etc. to estimate their own position. Then, in the second phase, the nodes utilize the position of the known anchors and the information obtained in the ranging phase to compute their own coordinates. The Cramer Rao Lower bound (CRLB) then defines the fundamental limit on the positioning accuracy of the nodes by modelling the impact of the noisy ToA, AoA, or RSS measurements on the ranging quality. Other bounds on accuracy, such as the Bayesian Cramer Rao bounds, the WeissWeinstein bound and the extended Zik-Zakai bound can be tighter and more informative than the CRLB when the localization system is map-aware. These bounds indicate that an accuracy of $2 \mathrm{~m}$ could be reached if a map-based priori knowledge and map-aware localization is used [16][17]. For instance, in [17], RSS based algorithms were evaluated using trace-driven analysis and shown to benefit from the addition of more resources up to a point beyond which their performance degraded. This effect was then mitigated by "cleaning" the data to remove "low quality landmark" where the quality is defined in terms of the fit of the distance to RSS model. Hence, the performance is enhanced by "cleaning" the data. The accuracies in order of $0.2 \mathrm{~m}$ are possible when utilizing commodity hardware. Additionally, the maximum error achieved in the worst case scenario can be decreased to reach $1.6 \mathrm{~m}$.

However, basic localization techniques have their limitations. ToA/TDoA is limited by the requirement of at least 3 base stations (or ANs) to generate 2-D fix. AoA requires at least two base stations. The performance of AoA techniques is highly dependent on the range giving significant position estimation errors from relatively small error in the AoA measurements. They are restricted by the carrier frequency, and the size of the array. Thus, they are used only for localization in applications with requirement of low accuracy or in combination with other measurements. Also, AoA systems are sensitive to angular multipath, a major effect in indoor environment. Consequently, ToA techniques are preferred in urban areas due to multipath effect whereas AoA are preferred in open areas.

Looking at the different access technologies for localization purposes, we can also note various limitations. For instance, empirical analysis of the appropriateness of WLAN localization showed that significant errors always occur, even though reasonable accuracy may be achieved [18]. Errors are mainly due to the presence of different locations with similar radio signatures, such as fingerprints or received signal strength, caused by the dynamic propagation of radio signals [15]. Thus, this is considered as a fundamental limit of pure WLAN-based techniques where large errors in range of 6 to $8 \mathrm{~m}$ occur. 
To give more insights about these aspects and limits, we will describe with the necessary details the stand-alone localization techniques used for indoor scenarios.

\section{BASIC POSITIONING TECHNIQUES IN INDOOR ENVIONMENTS}

Localization methods are based on the estimation of distance to anchor nodes with known positions and on internode measurements. Node cooperation enhances position estimation and is mostly beneficial when traditional localization techniques fail to produce accurate estimation, as is the case in indoor scenarios.

Linear least squares (LLS) lateration is a simple method for position estimation. Ideally, the unknown node would be located at the intersection of at least three circles with centers at the anchor nodes and radii equal to the distance to each of these anchor nodes. However, as it is highly unlikely that a single point of intersection is obtained, least squares (LS) optimization is used to minimize the sum of squared residuals. Consequently, the problem becomes a nonlinear optimization that needs proper initial estimates [19]. Since nonlinear optimization is computationally expensive, alternative methods, such as linearized expressions, are used to estimate the position using LLS. Although this is not an optimal solution for position estimation, it nevertheless achieves roughly good accuracy with low complexity [19].

In the following, we briefly describe basic standalone positioning techniques used in the context of homogeneous networks with the possibility of hybridization.

\section{A. Time of Arrival (ToA)}

The ToA approach includes the calculation of the time needed by the signal to travel from the UD to the ANs. The UD is localized to a circle centered on the AN with a radius $d$ estimated through the ToA. Hence, to detect the exact location of the UD, at least three ANs are required. In this case, the estimated position of the UD is simply within the region of intersection (if it exists) of the three circles, as shown in Figure 3. The actual estimated position could then be easily obtained through any filtering technique such as LS or Weighted Least Square (WLS) [20][21].

\section{B. Time Difference of Arrival (TDoA)}

TDoA examines the time difference at which the signal arrives at many measuring units. The transmitter must lie on a hyperboloid for each TDoA measurement with a constant range difference between the two measuring units. Such measurements are taken between multiple pairs of reference points with known locations. Also, relative time measurements are used at each receiving node in place of absolute time measurements. No synchronized time source is needed by TDoA to perform localization; however, synchronization is only needed at the receivers. The location to be estimated is the intersection of many hyperbolic curves, as shown in Figure 4. This technique is referred to as multilateration.

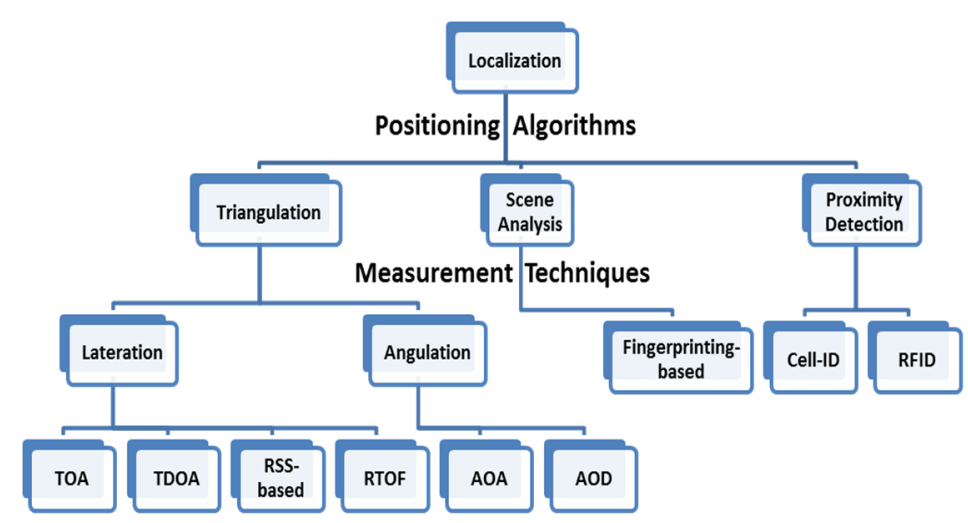

Figure 2. Classification of localization methods

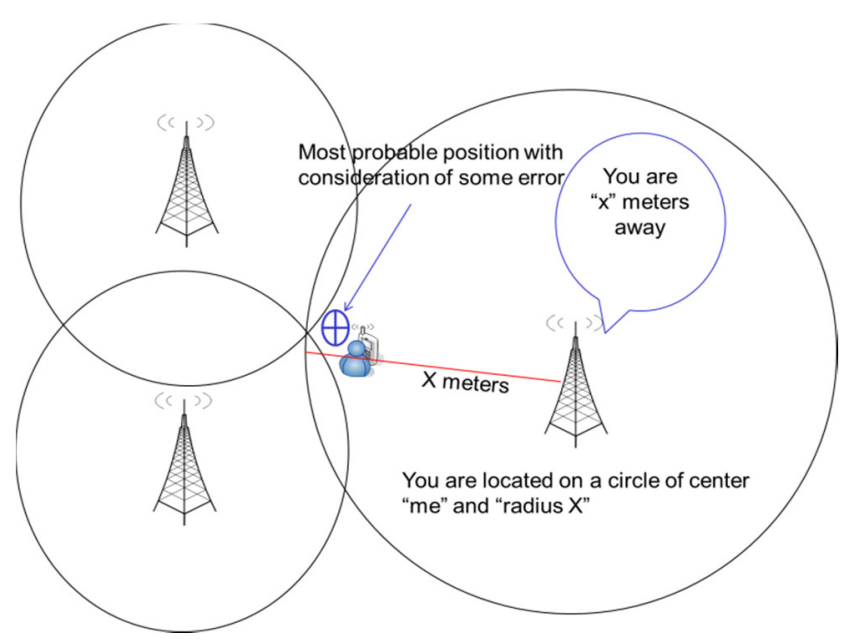

Figure 3. ToA Ranging

\section{RSS based Fingerprinting}

The RSS approach includes two main methods: the path loss lognormal shadowing model to deduce a trilateration, and the RSS fingerprinting [7]. The first approach is used to estimate the distance between the serving BS and the UD based on a path loss lognormal shadowing model, as shown in Figure 5. Then, trilateration is used to estimate the location of the UD using at least 3 serving BSs. On the other hand, the RSS-based fingerprinting firstly collects RSS fingerprints of a scene, as shown in Figure 6, and then estimates the location of the UD by matching on-line measurements with the closest possible location that corresponds to measurements in a database [4]. Therefore for each possible location, ambiguity points could exist leading to high estimation errors in standalone positioning scenarios.

\section{Angle of Arrival (AoA)}

This technique includes the calculation of the angle at which the signal arrives from the UD to the ANs. Then, the region where the UD could exist can be drawn, as shown in Figure 7. Basically, this region is a line having a certain angle with the ANs. Although at least two ANs are needed to estimate the location of the UD, the position estimation error could be large if a small error occurs in the AoA estimation. Therefore, the AoA based technique is of limited interest for positioning purposes, unless it is used with large antenna arrays. 


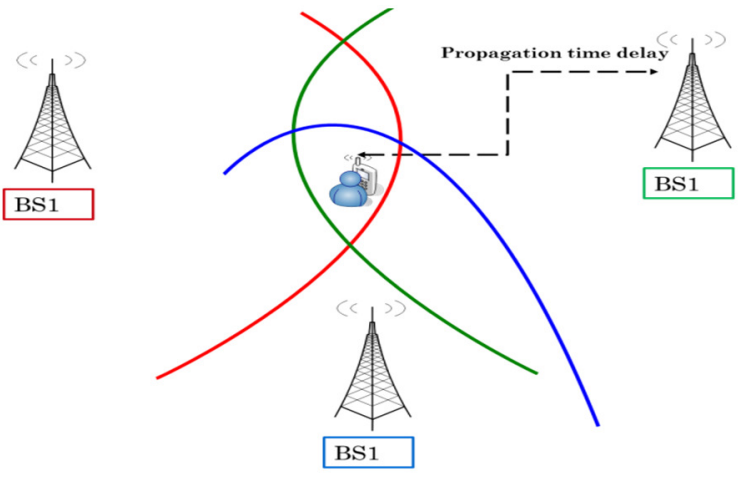

Figure 4. TDoA

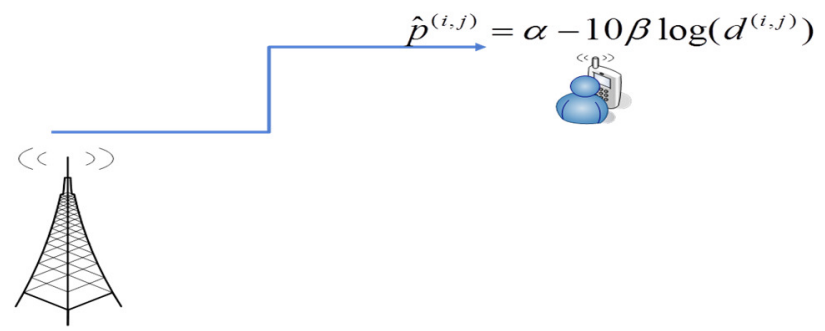

Figure 5. RSS for distance estimation

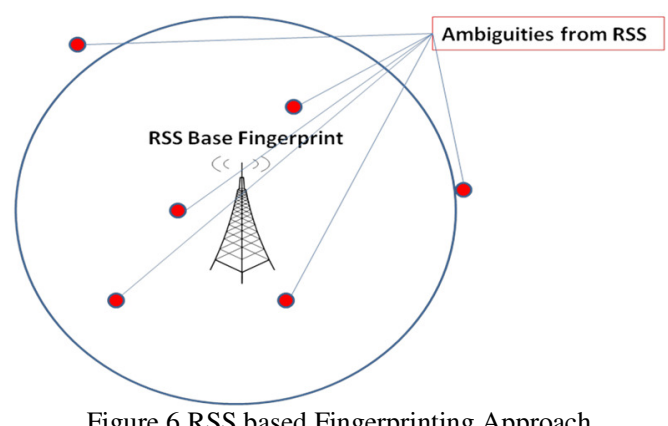

Figure 6 RSS based Fingerprinting Approach

\section{E. Hybrid techniques}

Recently, hybrid and cooperative mobile positioning has emerged as a new stream of wireless location; the core idea of cooperative positioning relies on the utilization of trustworthy short-range measurements to enhance the accuracy of the location estimation of a wireless system.

Different combinations of the basic standalone positioning techniques (RSS, ToA, TDoA, AoA, etc) have been implemented to enhance the accuracy of location estimation. For instance, a combination of ToA, AoA and RSS based fingerprint approach, as shown in Figure 8, provides initial estimation of UDs [22]. The hybrid ToA/TDoA and RSS proposed in [23] achieves further enhancement in terms of location estimation accuracy when compared to the use ToA or TDoA alone.

\section{F. Common Pitfalls in stand-alone positioning techniques}

Generally, stand-alone positioning techniques suffer from drawbacks affecting the localization accuracy. For instance, the ToA technique requires accurate timing reference at the UD and synchronization between this reference and the clock at the anchor. Obviously, it is highly challenging to achieve this task; if it is achieved, it will cause an increase in the cost and dimensions of the mobile device. Besides, a remarkable change in the software of the mobile device is needed by the TDoA approach along with further hardware installations. TDoA requires having the processing done at the UD and sending the estimated location to the system on the reverse link. Hence, the bulk and the costs of the handset will be increased in order to satisfy the estimation and synchronization needs. As well, the RSS technique has drawbacks in terms of difficulty to have a LOS between transmitter and receiver in indoor scenarios. Hence, localization accuracy is affected by the multipath effect induced in indoor environments. Above and beyond, pathloss models are used also to perform localization. However, shadowing and multipath fading effects deteriorate the accuracy of such models. The accuracy in this case can be improved by using pre-measured RSS contours centered at the receiver, or using many measurements taken at several BSs. Also, the enhancement of the localization accuracy can be achieved by using RSS measurement based on a fuzzy logic algorithm. As well, the AoA technique suffers from drawbacks such as complexity in terms of hardware requirements and reduction in the localization accuracy as the UD moves away from the measuring units.

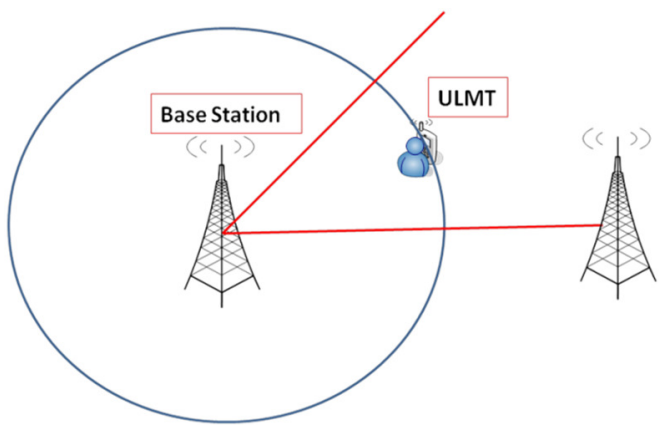

Figure 7. AoA Measurements

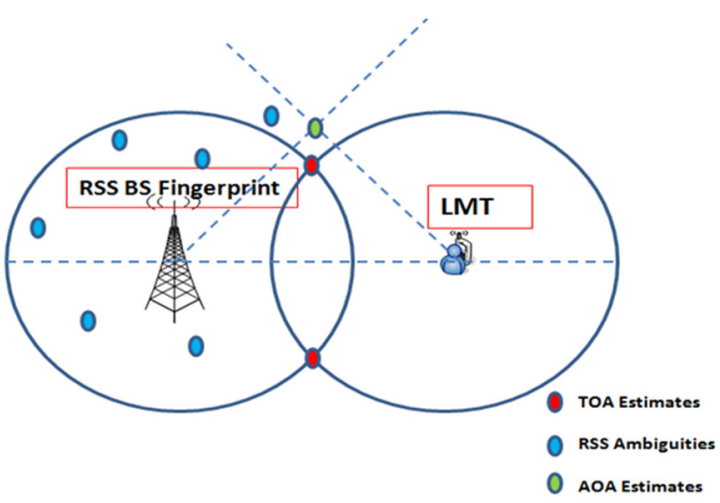

Figure 8. Combination of ToA, AoA and RSS fingerprints

\section{SYSTEM BASED LOCALIZATION IN INDOOR ENVIRONMENTS}

Despite the fact that hybridization of basic positioning techniques provides improvement in the localization accuracy, there is always a need nowadays for better methodologies that achieve enhancements in energy consumption and further improvements in accuracy. In the literature, different localizations techniques have been proposed that can be 
classified into the categories shown in Figure 2. In all these categories, research has been focused on advanced techniques based on new metrics and signals such as energy driven, signals of opportunity (SoOP), Ultra-Wide Band (UWB) in addition to the conventional systems such as WLAN, WSN, etc. So, we will discuss more intelligent positioning techniques; specifically, the UWB based localization, WLAN-based localization, and Sensors-based localization. Finally, we will discuss in this section the use of SoOPs as emerging technology to improve localization followed by the challenges of the system-based localization techniques in terms of energy and accuracy.

\section{A. Ultra-Wideband (UWB) based Localization}

UWB technology is based on transmitting ultrashort pulses less than $1 \mathrm{~ns}$, with a low duty cycle from 1 to 1000 . The transmitted signal in UWB is sent over multiple frequencies band. UWB then allows accurate localization and tracking of mobile nodes in indoor environments.

UWB technology is commonly used by researchers and industry in various fields such as indoor positioning in order to present enhancements in terms of achieving high range measurement resolution and accuracy, low probability of interception, multipath immunity, and the ability to combine positioning and data communication in one system. Additionally, UWB technology is highly scalable and can be used at low cost with a low energy consumption [24].

The position is estimated in UWB-based applications from the radio signals traveling between target node and reference nodes whose positions are well known. This procedure is done using ToA, AoA, RSS, TDoA, and hybrid technique as stated in Section III. Localization systems based on UWB technology achieve an accuracy of centimeters. With three-dimensional positioning based on ToA or TDoA, an accuracy of $15 \mathrm{~cm}$ in indoor scenario has been achieved [25]. Indeed, multipath signal components are decomposed by UWB receivers because they possess high bandwidth. In scenarios satisfying Line Of Sight (LOS) conditions, the first path that refers to the LOS component is the robust path used for localization purposes. Nevertheless, more complex techniques are needed if this is not the case in order to perform accurate estimation of the initial delay.

Furthermore, the time-based positioning techniques offer higher localization accuracy compared to RSS technique in UWB-based positioning applications since the high bandwidth of UWB is not efficiently used by RSS technique in terms of enhancing positioning accuracy compared to the time-based techniques. On the other hand, ToA and TDoA make use of the fact that UWB signal has high time resolution to increase the positioning accuracy relative to other techniques [26].

\section{B. WLAN-based localization}

WLAN is the most known solution for indoor positioning [11][12]. Positioning systems based on WLAN provide better performance compared to some technologies, such as GPS, global system for mobile communications (GSM) and Bluetooth. This aspect is due to the fact that WLAN positioning systems do not require any additional software or hardware manipulation, but are able to perform localization based on the existing infrastructure. RSS is the most known WLAN localization technique due its easy extraction in 802.11 networks and its ability to run on of-the-shelf WLAN hardware. On the other hand, ToA, TDoA, and AoA are less common to WLAN-based positioning systems since angular and time delay measurements are complex.

Usually, WLAN scanning aims to find available networks for connection. Typically, the scanning can be performed at low rate since the set of available networks changes slowly. When a device aims to estimate its own position while acquiring WLAN signals, recurrent RSS measurements are needed from the APs in order to minimize positioning error. A regular update is needed when a positioning device is moving along a trajectory; hence, scanning for available APs on all relevant channels is performed by each device at rate equivalent to the update rate. As averaging a set of RSS measurements reduces the effect of noise, a device that is concerned about positioning accuracy performs the scanning at a rate higher than the update rate. Alternatively, a slower scanning rate than the update rate leads to reduced power consumption at the expense of positioning accuracy. Hence, balancing the trade-off between power consumption and positioning performance is the main driver for a device in selecting its parameters for scanning in WLAN.

Two main approaches are used for indoor WLAN: monoobjective approach and multi-objective approach [27]. Both approaches are based on Variable Neighborhood Search, aiming to reduce the positioning error during WLAN planning process. The combination between WLAN positioning system and sensors embedded on smart devices obtains precise indoor localization for mobile smart devices [28]. The reader may refer to [8]-[12] for more information.

\section{Sensors Based localization}

Inertial sensors such as gyroscopes are widely used in localization. Nevertheless, inertial sensors, usually based on low-cost MicroElectroMechanical System (MEMS), suffer from errors which in turn affect the localization accuracy [29][30]. In order to alleviate this problem, the work in [31] employs "denoising", which reduces noise from raw sensor signals. This approach enhances the accuracy and performance of the system by avoiding breakdowns induced by excessive noise.

The localization in sensor-based technologies can be also divided into two categories: (1) Signature-based and (2) Beacon-based techniques. Signature-based techniques [32] assume non-uniform distribution of the nodes to be used as a signature for estimating location by noticing node neighborhoods. In Beacon-based techniques [33], Beacon nodes should identify their absolute positions using GPS or manual configuration. The location of the remaining nodes is estimated using distance/angle measurements to beacon nodes followed by multilateration or triangulation.

Sensors based localization is an important feature of mobile systems, such as Autonomous navigation, entertainment robots, service robots, and military robots [29][34][35]. In these 
environments, the localization of mobile nodes can be achieved using dead reckoning-based odometry through, for instance, wheel speed sensors [29],[34][35]. Emergency Sensor Networks (ESN) in contexts such as forest fires, natural calamities (hurricanes, storm), and terrorist attacks [36] are also of great interest. The placement of the sensor nodes in emergency applications is extremely localized for each point at which the size of the node group is based on the intensity of the monitored event at that point.

\section{Signals of opportunities (SoOPs)}

Currently, mobile terminals implement a variety of communications standards like GSM, UMTS, LTE or even short range communications like Bluetooth or WLAN. Therefore, such terminals are capable of listening to a broad spectrum of radio signals. The transmitters of such radio signals compare to landmarks in classical navigation. Their position might be known. But if the position of the origin of such SoOP is unknown, their observation can still potentially improve the positioning performance of classical mobile radio positioning using downlink reference signals for instance. Basically, these signals are not specific for navigation. SoOPs are RF signals utilized for communication purposes. SoOP has many advantages in terms of signal space diversity and higher received signal power.

Related work considers digital terrestrial video broadcasting signals for positioning purposes [37] based on signal propagation delay estimation. Here the transmitters are fixed and it is reasonable to assume their positions to be known. Skyhook has developed a positioning system based on WLAN signals [38]. This approach requires a database which must be learned and kept up to date using appropriate self-learning algorithms. So inherently this approach does not assume knowledge about the WLAN transmitter positions from the beginning. TV and WLAN signals are combined to improve positioning [39].

Moreover, different mitigation methods such as assisted GPS and differential GPS were proposed to provide enhancements to achieve robust navigation under critical scenarios. Recent proposed navigation systems use SoOP to enhance the accuracy. For instance, cooperative network is developed to provide accurate localization by using large number of nodes combined with SoOP [40]. The fundamental ability of cooperative network is that each node can produce navigation signals that can be used by other uses in the network in addition to receiving SoOP from selected towers. In such case, both local and global positioning is possible. Actually, the known positions of the SoOP provide global positioning. On the other hand, local positioning permits signals produced through the cooperative network. Thus, a SoOP technique can be used for the purpose of localization, such as using timing measurements to perform opportunistic positioning [41]. Many SoOPs are used for mobile localization without GPS [42][43].

\section{E. Challenges and Pitfalls}

The target of this section is to detail the main challenges and pitfalls of each system.

\section{1) Challenges in UWB-based localization system}

The performance of positioning systems based on UWB technology may face many challenges induced by aspects such as extremely cluttered operational environments causing multipath, NLOS and shadowing artifacts. For instance, in ToA-UWB based schemes and NLOS conditions, the performance degradation is mainly due to the mismatch between the first arriving path and the direct path and the addition of detouring delay. Another big challenge of UWB based localization resides in implementing wideband radio devices for a UWB signal with absolute bandwidth larger than $500 \mathrm{MHz}$. Here, some efforts have been done in the research community to develop such platforms reaching 10 to $15 \mathrm{~cm}$ in positioning accuracy. The reader might refer to [44] for more details.

In UWB-based positioning system, interference with the ultrawide spectrum may occur because of the misconfiguration. Interference may occur also due to the spread of the UWB signal over the bandwidths containing the frequency of the existing narrowband system. Another challenge resides in the need of, at least three receivers with unblocked direct path to the transmitter for normal ToA positioning algorithm. UWBbased positioning system requires also signal acquisition, tracking, and synchronization to be performed with very high precision in time relative to pulse rate. Currently, researchers are working on such problems. We can notice for instance the work in [45][46] where a novel technique for ToA with two receivers is proposed. Even though this work is very interesting to solve such issues, the door is still open for more solutions as limitations and challenges are still there.

2) Challenges in WLAN-based localization system

WLAN-based localization system is time consuming for site surveying and is labor intensive. Another challenge is the fact that the multipath of such systems is influenced by the existence of physical objects. Also, WLAN-based localization system may interfere with other applications in the $2.4 \mathrm{GHz}$ ISM. Moreover, the variation of signal strength with respect to time is considered a weakness of such system causing deterioration in the localization accuracy. The variation of signal strength caused by the movement of people, doors, and furniture in offices requires updating simultaneously the signal strength map. Hence, this is considered as a main drawback of WLAN fingerprinting systems.

\section{3) Challenges in Sensors-based localization system} In terms of methodologies, anchor-based localization techniques are usually preferred in this environment due to their accuracy. Nevertheless, such techniques have disadvantages, such as the need for proper anchors to be installed in WSN. As the anchors have high cost and energy consumption, the number of required anchors must be minimized. Another weakness of anchor-based localization techniques is that a uniform distribution of anchors is needed knowing that this criterion cannot be satisfied in many environments such as battlefields and natural disasters environment, where sensor nodes are deployed randomly. While localization of unknown nodes in WSN is done using randomly selected anchors, the anchors differ in their impacts on the accuracy of localization due to their characteristics and the uncertainties of wireless 
communication. To solve this problem, the work in [48] proposed a new anchor-based positioning technique by the creation of a database for optimized anchors. Then, anchors from the created database are used to measure the distance to the unknown node and the new located unknown nodes become new anchors in order to decrease the dependency of localization technique on anchors and to ensure that the anchors are uniformly distributed over the network.

\section{4) Common Pitfalls in SoOPs}

When using SoOP for the purpose of localization, one has to consider significant aspects such as the lack of independent SoOP, 2-D vs. 3-D solutions, SoOP clock errors, signal integrity, and multipath and NLOS signals. The uncertainty in the clock of the signal transmitter due to an offset relative to the clock of the receiver in addition to the unknown transmission time is a challenge against localization. As well, hybrid/fused SoOPs are used in order to alleviate the technical challenges obtained from positioning with SoOP [42].

\section{5) Energy efficiency: A common challenge}

In many indoor scenarios, the positioning techniques have to be energy efficient as they are based on devices with limited battery life such as sensors, smartphones, etc. Nevertheless, these positioning techniques have various criteria of energy efficiency, service availability, and accuracy. For instance, the energy used during the idle state by an access point (AP) in WLAN networks is wasted if no user is being served. As stated in [49], more than $\$ 6$ billion is spent for almost 74TWh of electricity consumed for internet related equipment every year in USA. Hence, there is a vast need for energy efficient positioning techniques.

Although the WLAN based positioning system service approaches are energy efficient techniques, they are not generally obtainable for users. Hence, the work in [50] proposed to use the current WLAN infrastructure and Access Points (Aps) without pre-deployment calibration and a genetic algorithm for energy-efficient localization purposes, like WLAN access points (APs). Consequently, this approach is restricted to indoor scenarios.

Another interesting approach resides in power cycling algorithms in smartphones [51]. The duty cycling approach polls the built-in sensors of a smartphone at specific time intervals to identify any mobility and whether to turn the GPS on or off. A Bluetooth-based Position Synchronization (BPS) is another idea of energy efficient positioning technique [51]. The location information is shared among devices using BPS over a Bluetooth connection.

Furthermore, positioning systems based on the combination of different methodologies and techniques are also energy efficient. Here we distinguish the works of [52] which provides location estimates through the utilization of heterogeneous positioning services and the combination of techniques including a received signal strength indicator and a 2D trilateration, and of [53] based on context information and a fusion engine using particle filters. Moreover, we provide the work of [54] which introduced a preliminary analysis of probabilistic localization techniques for power-efficient mapaided localization, developing the green global-greedy position estimation (3GPE), and presenting entropy deduction as a new metric for performance assessment. However, this approach still has many challenges. The first is the determination of the inherent relations between the expected errors, the location precision and the probability of each possible matched fingerprint. The second is the evaluation of the accuracy contribution of each AP using the entropy deduction metric of probabilistic fingerprint [55][56].

When accuracy and energy efficiency are required, more technologies advancement should be achieved. This can be obtained by making use of hybrid data fusion concept and cooperative approach between different localization systems as discussed in section $\mathrm{V}$.

\section{COOPERATIVE LOCALIZATION AND HYBRID DATA FUSION}

\section{A. Hybrid Data Fusion (HDF)}

So far, heterogeneous wireless resources are included in most of wireless environments, such as LTE femto base stations, WLAN APs and WSNs. Also, such environments are characterized by the crowded cooperation over medium or short ranges between multi-standard UDs. Additionally, radiolocation ability is efficient in such scenarios for the sake of enhancing connectivity performance and enabling contextbased services or indoor navigation [57].

However, due to the signal attenuation and the multi-path propagation problems caused by reflections of radio signals, the accuracy of wireless-based indoor positioning is severely degrading in uncontrolled environments. Many researchers combine other sources of sensors (e.g. inertial measurement unit (IMU), cameras, and range finders) to compensate for these problems. Fusing the information from different positioning systems with different physical principles can improve the accuracy and robustness of the overall system.

It is worth noting that various wireless devices, such as Bluetooth, WLAN and radio frequency identification (RFID), provide a localization accuracy ranging from several meters to centimeters inside buildings. So, we will review in this section first the conventional techniques based on HDF. Then, we will discuss the fusion with maps and fingerprinting, fusion with inertial information, fusion with camera information, and fusion with other information such as the spatial structure information of an environment.

\section{1) Conventional HDF Techniques}

Recently, research work has been focusing on two main approaches in HDF, the centralized and non-centralized approaches. Iterative positioning (e.g. [58],[59],[60]) and cooperative links selection (e.g. [61],[62]) are used with the non-centralized approach. Moreover, such heterogeneous and cooperative environments include complex phenomena such as the conjunction of harmful sparse connectivity, space-time correlations among various radio access technologies and poor Geometric Dilution Of Precision (GDOP) conditions. The authors of [63], however, carried out extensive research that dealt uniquely with cooperation in homogeneous scenarios. As well, a measurement campaign in jointly heterogeneous and 
cooperative wireless indoor scenarios uses ZigBee devices with RSS measurement abilities [64].

HDF methods estimate reliable position information to the benefit of communications. The work in [65] has introduced the main radio technologies and scenarios for wireless positioning assessed by the "Wireless Hybrid Enhanced Mobile Radio Estimators (WHERE)" European project. Two novel particle filter based HDF techniques are used to either track the position directly from the received signal or the path dependent parameters. Moreover, new cooperative schemes, based on obtaining proper mathematical representations corresponding to LOS and non-LOS propagation, are used to enhance the performance of mobile communication systems in terms of position accuracy and reliability.

Another interesting set of work resides in the combination between angle-based localization, map filtering, and pedestrian dead reckoning [66] where absolute location estimates are provided by the angle-based localization system. On the other hand, accurate length and shape of the traversed route are obtained from pedestrian dead reckoning (PDR) without absolute location and heading information. The estimates obtained from PDR movement and angle-based location techniques are merged together with a building vector map in a particle filter that is used as the fusion filter in this study. Hence, merging information from different positioning techniques can lead to higher positioning accuracy for several of indoor scenarios.

Hybridization is also used for the purpose of pedestrian tracking [67]. Usually, this hybrid technique merges inertial measurements and RSS information via a Kalman filter. Classic hybrid methods for pedestrian tracking (e.g. [68] and [69]) were based on the utilization of a map-based or fingerprinting RSS localization method. However, this requires a time-consuming calibration step in order to create the radio map of the environment to be utilized for position estimation of mobile node through the matching between map measurements and mobile's measurements. On the other hand, another hybrid localization method [67] uses a channel modeling technique where a propagation channel model serves to give a direct relation between the distance between two nodes and the RSS; then, a positioning technique or triangulation is utilized to estimate node position from a set of distances to some known anchor nodes. Yet, fingerprinting methods provide higher accuracy; however, this approach has minimal calibration cost. Additionally, the fusion between inertial measurements and channel-based localization provides enhancements over fingerprinting methods in terms of positioning accuracy [67]. Generally, techniques based on LS are mainly used in cooperative positioning schemes as in [70]-[73]. On the other hand, statistical approaches such as factor graphs [45] and belief propagation [74] make use of a set of observations and $a$ priori probability distributions of node positions to estimate the maximum a posteriori location.

It is worth mentioning that distributed localization is also used in HDF through the so-called iterative multilateration (see [75] for instance). Once the position is estimated for an unknown node, this node is used as an anchor node whose estimated position is broadcasted to all neighboring nodes. The procedure is shown in Figure 9, where the target can do self-localization by making use of not only neighboring anchor nodes, but also virtual anchor nodes that have been localized in earlier iterations. Virtual anchors are localized with different levels of uncertainties. Also, this process is iterated until all nodes with at least three reference nodes achieve estimation for their position. In this case, information is needed only within local neighborhood, thus reducing communication cost at the cost of error propagation. Consequently, it is essential to have careful selection of reference nodes in order to reduce the accumulation of error through considering the uncertainties in estimating reference nodes.

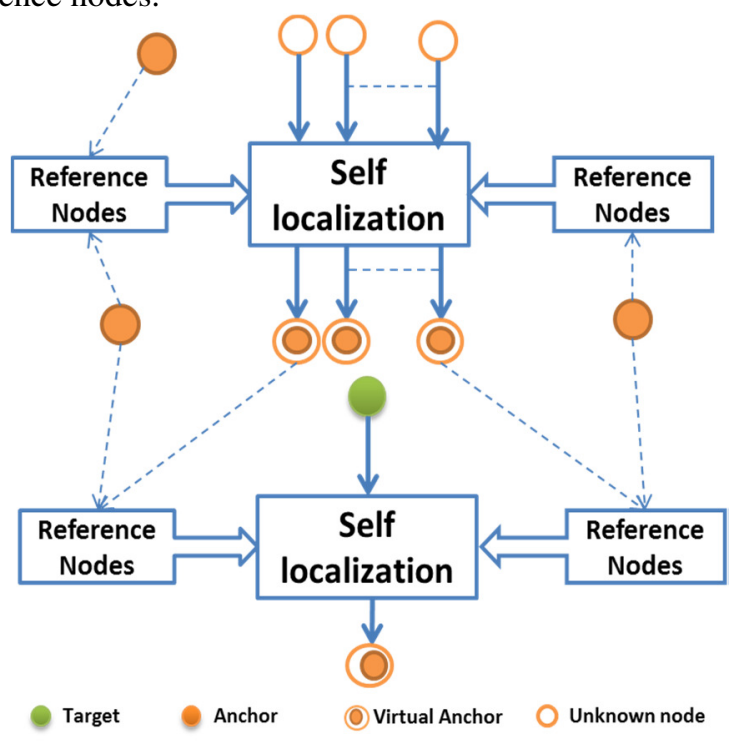

Figure 9. Iterative Multilateration

2) HDF Techniques including maps and fingerprinting A number of propagation model-based or fingerprinting-based techniques have been proposed for indoor wireless positioning. $\mathrm{RF}$ location fingerprinting [76] uses a set of sensor measurements (i.e. RSS) from WLAN access points, GSM, RFID readers, or other RF-based sensors to represent the locations. On the other hand, propagation model-based approaches [77][78] require an explicit sensor model to predict the propagation of the RF signals. However, their accuracy is affected by a large number of environmental factors, since it is almost impossible to find a universal model to characterize the radio signal propagations in indoor environments due to severe multipath and numerous site-specific parameters. This approach does not rely on any explicitly predicted model to characterize the sensor's behavior. Instead, an a priori set of fingerprints that expresses the sensor's output at sampled locations in the global frame has to be recorded in advance during an offline training phase. In the online localization phase, the location of a node is determined by matching the current observations with the recorded reference fingerprints. Fingerprinting-based approaches are therefore assumed to be more accurate and robust as compared to the model-based approaches with regard to location-specific distortions.

The traditional fingerprinting-based approaches require a timeconsuming and laborious site survey phase in order to construct, combine with and update the fingerprinting map. Therefore, 
some researchers proposed different techniques to reduce the site survey in the traditional fingerprinting-based approaches. Here, we mention the works of [79] exploiting human motions, [80] using smartphone-based crowdsourcing, and [81] adopting ray tracing tool.

3) Fuse with inertial information

The movement of a mobile object can be estimated from the IMU and thus can be integrated into indoor positioning systems to improve their performance. The position estimates can be improved and smoothed by fusing the WLAN and IMU data [82]. Moreover, the orientation information can be retrieved using the movements of the pedestrian, which leads to a more precise WLAN positioning system. The IMU information assists the selection of nearest neighbors for real-time WLAN fingerprinting positioning in order to reduce the positioning error [83]. It is combined also with a region-based fingerprinting using a Kalman Filter to improve the positioning accuracy [84]. The accuracy is further improved through a multisensory system, which combines data from different sensors (RSS, visual features, and built-in accelerometer) in smartphones [85].

4) Fuse with Camera information

A hybrid indoor location estimation system is achieved also by merging the information from WLAN with the build-in camera on a smart-phone for position estimation [86]. This approach utilizes visual markers pre-installed on the floor for the position correction. Visual information is combined also with the radio data to track a person wearing a tag using a mobile robot in indoor environments [87]. The authors of [88] presented a method to integrate range-based sensors and ID sensors (i.e. infrared or ultrasound badge sensors) using a particle filter to track people in a networked sensor environment. As a result, their approach is able to track people and determine their identities owing to the advantages of both sensors.

\section{5) Fuse with other information}

The spatial structure information of an environment may be used to improve the localization accuracy. Given a map of an environment, an object can only appear in the free space of the environment. Hence, different kinds of information fusion lead to an improvement in the positioning accuracy, usually at the cost of additional complexity. For instance, data fusion occurs also with different types of RF sensors to improve the localization accuracy since different positioning systems may complement each other [89].

\section{B. Cooperative Localization in SLAM}

The Simultaneous Localization And Mapping (SLAM) technique performs localization relative to a map of an unknown environment that is simultaneously acquired by a moving node. While SLAM techniques are used in outdoor environments, their utilization in indoor scenarios is however dominant. SLAM is a localization technique used to build up a map within a known or unknown environment while at the same time tracking the current location. SLAM algorithms are tailored to the available resources, aiming to work with operational compliance and not targeting perfect results. Published approaches are employed in self-driving cars, unmanned aerial vehicles, autonomous underwater vehicles, robots and even inside the human body. SLAM is mainly used in cases where nodes are not equipped with a GPS sensor. As alternative, incremental egomotion sensors, such as inertial navigation and odometry, are used for localizing the mobile node. Nevertheless, error is accumulated over time by such sensors making accurate map generation a challenging task.

In practice, we have two main methods listed under cooperative SLAM localization. The first one is the centralized approach through which a central system distributes the data to all nodes (such as robots) in the group. The weakness of this system is that any fault in the central unit leads to a failure of the whole localization system, and nodes are limited in mobility in order to keep contact with the central system. On the other hand, the second method is the decentralized approach through which the exchange of the data between nodes is done without the need of a central unit [90][91].

In SLAM, the data, of both the sensor networks and the autonomous nodes or vehicles, is usually merged for enhancing localization techniques [92]. Basically, the one-way cooperative localization scheme is based on the fact that one system is supplementing the other to do localization. The twoway cooperative localization technique is based on the idea of sensors performing their own localization by themselves first and then performing localization of the vehicles (or robots), while the localization of the vehicles will be done by themselves.

SLAM presents critical challenges in robot research community due to the non-linear nature of the problems therein. As discussed by [93], Extended Kalman filter (EKF) is widely utilized for the localization of robot and the incremental development of the environment map in SLAM. Besides, the localization in non-linear SLAM systems is tackled also in EKF literature. Particle filter is also used to denote both possible map configurations and robot poses. Using a new map representation denoted by distributed particle mapping allows an efficient preservation and update of hundreds of robot positions and candidate maps.

Simultaneous Localization and Mapping and Moving Object Tracking (SLAMMOT) represent a normal continuation for the SLAM problem with moving objects. The SLAMMOT technique is used to solve the observability problem as well as enhance the accuracy of localization, mapping, and tracking [94]. Other extensions of SLAM techniques also exist. We notice the Cooperative Simultaneous Localization, Mapping and Target Tracking [95], the distributed strong tracking unscented particle filter which uses distributed particle filter [96]. All these techniques are applicable in indoor environment.

\section{Common Pitfalls to avoid}

In this section, we handle the pitfalls to avoid while using data fusion techniques in cooperative localization.

Data fusion is highly critical in WSN since it increases the network lifetime and achieves the objectives of the application, such as target tracking, event detection and decision making. Consequently, applying inappropriate data fusion leads to misleading evaluations and waste of resources. Hence, we must take care of probable limitations of data fusion so that we can 
prevent blundering cases from occurring. Also, WSN recommends applying data fusion in a distributed fashion in order to increase network lifetime. Nonetheless, we must also take care of the challenges obtained when performing data fusion in a distributed manner. Therefore, a centralized fusion system may provide better performance than the distributed system.

Moreover, the feasibility of data fusion techniques requires the evaluation of process computational cost and the delay presented in the communication. The centralized version is preferred when computational resources are available and the cost of transmission is somehow low.

Statistical data fusion techniques such as probabilistic data association and Kalman techniques have an optimal performance under particular conditions. However, the target can't be assumed to be moving independently and the measurements can't be assumed to have normal distribution around the estimated position. Second, it is difficult to attain $a$ priori probabilities for detection errors and misleading measurements, knowing that statistical methods represent events as probabilities. On the other hand, the complexity of statistical techniques optimizing numerous frames increases exponentially with the number of targets. Hence, such techniques are considered to be computationally intensive. So, it is preferred to assume no interaction between particles and to perform individual tracking.

Challenges are also induced by the data fusion process with distributed data fusion techniques. These challenges include out-of-sequence measurements, temporal and spatial alignments of the information and data correlation. As shown by many researchers [58]-[96], the cooperative and hybrid data fusion approaches enhance significantly the accuracy of localization and navigation of mobile users, despite of the drawbacks listed above. However, the accuracy limit is still not clear and requires further investigation. In philosophy, learning from your past evolves your future. As Leo Buscaglia said, "Change is the end result of all true learning".

\section{LEARNING ALGORITHMS FOR LOCALIZATION}

\section{A. Learning and environmental mapping}

In localization, both supervised and unsupervised learning approaches have been proposed. For instance, in SelfOrganizing Map (SOM), the adopted approach is unsupervised learning [97]. Nevertheless, supervised learning techniques are presented since the relationship from distances information (inputs) to desirable behavior of the node (outputs) to be localized are achievable.

In SLAM, learning techniques are widely combined with environmental mapping. For instance, the standard SLAM is extended into signal-strength-based SLAM with learning approaches through the Gaussian Process Latent Variable Model (GP-LVM) [97]. Combining GP-LVM and a dynamic motion model, a topological graph of the environment is built up from the raw signal strength data. This map is then used for efficient localization by applying Bayesian filtering [98][99]. In indoor environment, learning and radio maps are used to obtain high accuracy. Radio map can be automatically constructed via crowdsourcing and RSS measurements [100][102]. However, as RSS measurements are vulnerable to environment dynamics they are more extravagant in indoor environments with severe multipath. Consequently, the location will be estimated with low accuracy. Doing the site survey repetitively and applying appropriate learning approaches solve this problem despite of its complexity in terms of time consumption. Fresh RSS measurements can be taken to adapt the radio map by deploying fixed reference ANs [103]-[106]. Hence, location service is enhanced with high quality when the radio map is always updated through learning. Among others, game theory has gained a lot of research interest in this domain.

\section{B. Game Theory to improve Localization}

Game theory originates from the field of applied mathematics to analyze complex interactions between entities. Basically, game theory is a set of analytical tools that perform distributed decision process. Generally, a game is composed of decision makers, i.e. players with different strategies. Each player has a utility (payoff) that represents the level of satisfaction. The aim of each player is to maximize his own expected payoff [107]. Game theory includes coalition formation. The classification of coalition game is done by mapping a network component as a game component, nodes as players, available adaptations as action set, and performance metrics as utility function [108].

1) Game Theory in localization

Game theory is very efficient for localization purposes since it permits the formation of optimal coalitions of nodes for localizing a target node. Greedy strategies are usually proposed and used to minimize the error of their localization process and reduce the power consumption. Thus, it is important to study the tradeoffs in selecting an effective strategy for indoor localization.

2) Coalition Games for positioning purposes

Game theory has been used in localization, primarily for demonstrating the tradeoff between cost and performance and for selection of reference nodes [109], specifically for allocating measurements between reference nodes aiming to localize the target. In a distributed localization approach, the concept of game theory and utility functions is used to determine the combination of reference (anchor) nodes that lead to the best localization performance [75]. To implement the cooperative localization approach, a coalition game denoted by the pair $(N, v)$ is adopted, where $N$ represents a finite set of players and $v$ denotes its utility function. The players are the element of $N$ and any non-empty subset $C \subseteq N$ represents a coalition, as shown in Figure 10. Particularly, $N$ represents the grand coalition. Basically, the coalition value, represented by $v$, denotes the value of a coalition in a game. The payoff $x_{i}$ of each player $i \in S$ represents the amount of utility received by each player from the division $v(s)$. Hence, the payoff allocation is denoted by $x \in \mathbb{R}^{|S|}$, where $|S|$ is the cardinality of $S$.

The localization procedure here is demonstrated as a game fitting in the class of weighted-graph games. For such illustration, the players are defined as the vertices, and the value of a coalition can be defined as the summation of the weights of edges connecting pair of vertices in coalition. The main 
concept is to allocate more measurements to nodes with higher contribution in the localization process.
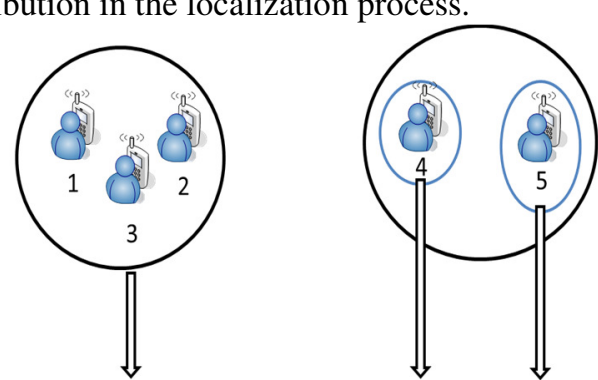

Coalition $S_{1}=\{1,2,3\}$

Coalition $S_{3}=\{4\} \quad$ Coalition $S_{2}=\{5\}$

Figure 10. Coalition Games

Generally, coalitional games are categorized into two kinds depending on the distribution of the gain among players in a coalition. The first type is the transferable utility (TU) game where we may have proportionality in the total gain attained between players in a coalition bound by feasibility constraints. A non-transferable utility (NTU) game is the second type. In such game, additional constraints, preventing arbitrary allocation, are imposed on the assigned strategies. Also, the payoff of each player is based on joint actions inside coalition. The localization problem can be also seen as a coalitional formation game within nearby anchors [110], where coalition formation is done through merge and split procedure, to reduce the cost of communications. The localization problem here is a game of NTU nature since the same position estimate is obtained by all nodes within the locating coalition and the power consumed is determined based on the relative locations of the nodes. To do so, a utility function is defined to model the cost and quality indicator of the localization [75]. In practice the definition of the cost and utility functions depend on the coalition cardinality, the limit of error targeted and the range of distances to be estimated through coalition games. For instance, a good choice is to define a utility function which increases with the distance between the anchor nodes and/or the error between the estimation and real measurement decreases. In such a choice is made, the cost of coalition will be less. In a real scenario with multiple anchor nodes, the following utility function of a node $x$ could be made:

$$
f_{x}(N)=\alpha\left[1-\frac{1}{N} \sum_{i \in N} \frac{\left(d_{i}^{\prime}-\hat{d}_{i}\right)^{2}}{R^{2}}\right]-\frac{(1-\alpha)}{N} \sum_{i \in N} \frac{d_{i h}^{2}}{R^{2}}
$$

where $0 \leq \alpha \leq 1$ regulates the tradeoff between cost and performance, $N$ is the number of measurements taken at a given location $x, d_{i}^{\prime}$ represents the estimated distance of the $i$-th node, $\hat{d}_{i}$ represents the distance between the $i$-th node and the joint estimated target position, $h$ is the coalition head index and $R$ is the coverage range.

One can also define other utility functions determined by locations of all nodes in the coalition with cardinality $N$ at a given measurement instance by:

$$
f(N)=\alpha\left[1-\frac{1}{N} \sum_{i \in N} \frac{\left(d_{i}^{\prime}-\hat{d}_{i}\right)^{2}}{R^{2}}\right]-\frac{(1-\alpha)}{N} \sum_{i \in N} \frac{d_{i h}^{2}}{R^{2}}
$$

The interest in each coalition function defined above is different. For instance, the first coalition could be used at each node at different time stamps to reduce the total energy consumption throughout all readings. The second function could be used with spatial correlation of nodes readings. A good example therein is given in [110] where the authors proposed non-super-additive coalition. It is the case when the grand coalition, a coalition that comprises all nodes, is not optimal.

Another approach in coalition games would be in considering the coverage area of the target node as a set of correlation regions [111]. The correlation region in this case represents the region where readings reported by nodes are alike. According to the localization performance, it is viable to assume that redundant information is provided by spatially correlated nodes in terms of GDOP or CRLB. Hence, we can represent the coverage area of the target node by set of active nodes acting as anchors. This approach reduces the complexity of the search method because only a subset of anchor nodes is involved in the process of forming coalition.

As a common rule of thumb, the localization in indoor environments from the perspective of a game theory deals with mobile devices as players competing on limited resources to perform position estimation [109]-[111]. This problem is formulated as a standard non-cooperative game, where the players are the mobile devices, the strategies are the scanning parameters and the payoff for each player involves the accuracy level of positioning and the power consumption.

\section{Open challenges in localization based learning}

In general, many challenges are recognized when using learning techniques for localization in heterogeneous wireless environment. In multi-agent localization, various activities are proposed to handle centralized localization approaches through the utilization of physical tagging. This helps in identifying the single agent position to global positioning systems based on cameras or GPS. However, in practical environments and mainly in indoor scenarios, it is preferred to avoid these centralized multi-agent approaches since they reduce the system autonomy and range of applicability. Based on that, probabilistic and distributed framework solves the problem of mutual localization with unidentified relative position measures [112][113]. This comes at the detriment of centralized failure risk.

In game theory based localization, a main challenge is to recognize the players and identify the localization problem as a cooperative or non-cooperative game. The selection of the players and strategies defined by each player with their objectives are vital in identifying the game. Players are assumed to be rational individuals in game theory since the actions taken by each player is based on his best interest. In such scenario, game theory models and explores the competitive or cooperative interaction among nodes (anchors or not) and/or network operators that represent rational decision makers.

Another challenge in cooperative games resides in the need of collaboration among users for the sake of maximizing their payoffs, in our case the location information; however, players may refuse cooperation and be selfish in order to preserve limited resources such as energy or optimize their own profit. Hence, incentive mechanisms are preferred to be embraced. Furthermore, the decision process done by the players is considered as a critical feature to consider in game theory in general and in localization particularly. 
Finally, in indoor wireless environment, defining the utility function as a function of the location is a complicated task due to the vast number of several applications with dissimilar needs.

\section{APPLICATIONS}

In this section, we detail the main applications of localization in indoor environments. Indoor positioning and navigation for mobile devices is a market with expected size of 4 billion $\$$ in 2018 [114]. A reliable, user-friendly, and accurate solution for indoor positioning and navigation might open the doors to the definition of new applications and the creation of new business opportunities in countless scenarios [115], and is indeed considered as a cornerstone in the realization of the Internet of Things vision [116][117]. It is worth mentioning that some of these applications could be also applied in outdoor scenarios.

\section{A. Robotics}

Robotics is one of the main applications of indoor localization. Many researches and developments have been conducted for implementing mobile multi-robot systems applications. The movement of robots in real large indoor scenarios where cooperation is required between them is a critical topic. For instance, cooperation between robot teams enhances the mission outcomes in applications such as surveillance, unknown zones exploration, guiding, or connectivity maintenance. Ubiquitous Networking Robotics in Urban Settings (URUS) project [118] is an excellent example using localization for evacuation in case of emergency where the robots lead the people to safe area via safe pathways in case of fire.

Moreover, obstacle avoidance and dynamic and kinematic constraints are considered in robotics in order to achieve complete navigation system [119][120]. Similarly, the work in [121] used the idea of forces among robots to avoid obstacles. Another application is seen in [122] where a multi-robot navigation system takes into account all robot (dynamic) constraints and calculates one function for all the formation to perform robot navigation with assured collision avoidance.

SLAM is also very interesting in robotics. Therein, the tasks performed by a robot are done autonomously without the awareness of its surrounding and own position. SLAM is useful in construction autonomous robots. However, it requires high processing resources to carry out SLAM in real time [123]. Then, C-SLAM [124] solves the problem by constructing the environment map based on the collaboration between multiple robots. A global map is obtained in C-SLAM by merging individual maps obtained by multiple robots.

Additionally, accurate navigation of mobile robot in indoor environments is achieved through an UWB navigation system [125]. The navigation system consists of two sub-systems: the robot control system and the localization system. Autonomous robot navigation is achieved in this system through a TDoAbased localization technique, digital implementation of transmitter and receiver and combination of both sub-systems [125]-[127].

\section{B. Ambient Assisted Living and Health Applications}

Indoor localization is one of the useful constituent in AAL tools. AAL environments are generated from "ambient intelligence" which is an advanced tool performing creative machine-human interactions. For instance, AAL tools aim to enhance health status of older adults by making them able to control their healthiness conditions [128][129]. Elderly people make use of such applications for the purpose of monitoring, tracking and other location-based applications. Besides, we realize the significance of such applications in indoor scenarios where GPS signals are useless. Some of indoor localization systems based on AAL applications are "Smart floor technology" to notice the existence of people [130] and "Passive Infrared (PIR) sensors" to notice motion of people. Currently, indoor positioning is central in the deployment of seamless emergency response services such as E911 in the US, hindered by the lack of common benchmarks and platforms [131][132]. This has recently pushed different research entities worldwide to propose advanced and beyond state-of-the-art solutions in this domain with applications varying from ambient assisted living, elderly support, emergency cases, etc. Here, we should mention the work of the project SALICE [133][134] which proposed solution based on the heterogeneous combination between satellite-based localization and communications systems to provide emergency services. The project is mainly based on combining delay diversity techniques and maximum ratio combining between satellite and terrestrial sites. The deployment of gap fillers (used as relays) on the border of the emergency area is another proposition of the project to increase the signal diversity by alternating between LOS and NLOS conditions.

In parallel, standardization bodies also recognized the fundamental role of location information, and are actively working on standards related to the retrieval of location information, the Location Working Group within the Open Mobile Alliance [135] and the technical specification TS23.271 by the 3GPP [136] being notable examples, as well as the upcoming 18305 standard by International Organization for Standardization and International Electrotechnical Commission, whose joint JTC1/SC31 subcommittee includes a working group on positioning. Last but not least, hardware companies are also pursuing the design of systems and chips for accurate indoor positioning, e.g. based on IEEE 802.11ac or IEEE 802.15.4.

Investigations in indoor scenarios on UWB for human body localization are performed via numerical and analytical methods by placing wearable compact sensors on the upper part of the body. This work succeeded in performing 3D localization using such sensors. Also, UWB technology is appropriate for wearable wireless sensor networks, and a 3D localization accuracy of $2 \mathrm{~cm}$ to $3 \mathrm{~cm}$ is achieved; hence, a system with this accuracy can be applicable for monitoring patient, tracking and applications for capturing motion.

Other applications are also based on UWB technology [47][137] where orthopedic computer-aided surgery as well as its integration with smart surgical tools such as wireless probe for real-time bone morphing is implemented [47]. UWB positioning 
system is proven to achieve a real time $3 \mathrm{D}$ dynamic accuracy of $5.24 \mathrm{~mm}$ to $6.37 \mathrm{~mm}$. Hence, this dynamic accuracy implies the potential for millimeter accuracy. This accuracy satisfies the requirement of $1 \mathrm{~mm}$ to $2 \mathrm{~mm} \mathrm{3D}$ accuracy for orthopedic surgical navigation systems.

\section{Location-based Services}

Location-based Services (LBS) are defined generally as service that outspreads GIS capabilities or spatial information to end users through wireless networks and/or the internet. Yet, LBS applications can offer the context and the connectivity needed to dynamically associate the position of a user to context-sensitive info about current environments; whereas conventional GIS applications are concentrated on geographical information for land planning and management. Hence, high level of personalization is achieved with LBS applications that simplify a capability of making each user the center of his universe.

LBS send data dependent on context and accessed by a mobile device by knowing the geographical location. LBS service is required for indoor and outdoor environments. For instance, indoor LBS include applications to obtain safety information or up-to-date data on cinemas, events or concerts in the vicinity. Further applications of this type include a navigation application used to direct a user to the target store in a public building. Moreover, LBS are used for advertisements, billing, and for personal navigation to guide guests of tradeshows to the targeted exposition booth. Also, LBS applications can be used at bus or train stations to navigate to the bus stop. Likewise, LBS are used for notification based on proximity, automated logon/logoff tasks in institutions and the profile matching.

\section{D. $5 G$ Networks}

Location information can be used to address many challenges in $5 \mathrm{G}$ networks [138]. The accuracy of location estimation was improved from hundreds to tens of meters using cell-ID localization in $2 \mathrm{G}$, localization based on timing via synchronization signals in $3 \mathrm{G}$ and reference signals dedicated for localization in 4G. As well, localization technologies can be used by numerous devices in $5 \mathrm{G}$ to attain an accuracy of location estimation in the range of centimeter. Basically, in 5G networks, it is expected to use precise location estimation through all layers of the communication protocol stack [139]. This fact is due to several aspects [138]. One of these aspects is the inverseproportionality between SNR and distance due to pathloss; hence, the distance becomes an indicator of the interference level and the received power. So, the best multi-hop path between the source and the destination in a dense network becomes the shortest path in terms of distance when neglecting the shadowing factor. Next, remarkable differences in the localized power are recognized due to shadowing effect. Additionally, nearby terminals can be used to induce local channel information due to the fact that shadowing often reveals decorrelation distances larger than localization uncertainty. So, the use of precise location information by $5 \mathrm{G}$ networks over all layers of communication protocol stack is due to the prediction of most of the $5 \mathrm{G}$ user terminals in their mobility patterns knowing that these terminals will be either associated with fixed or controllable units or people. Last but not least, localization is not only required for location-based services, but also for several jobs in cyber-physical systems, like smart transportation systems and robotics in 5G networks [140][141]. Furthermore, methods for resource allocation based on location awareness [142] can diminish delays and overheads since they can predict the quality of channel further than customary time scales. This is indeed one of the technical targets of the $5 \mathrm{G}$ technologies.

\section{COMPARATIVE STUDY}

In this section, we will present a comparative study between different localization techniques in terms of range accuracy for different applications. Basically, we present in Table 1 a comparison between different positioning systems showing the range, accuracy, and the technique(s) used. All of the positioning systems and the location information are reported from literature and produced in real time (according to the references mentioned).

Moreover, we can observe in Figure 11 the accuracy achieved by different wireless based positioning systems used for indoor, outdoor and locally urban, and rural and remote areas. It is very clear from this figure that the accuracy requirements depend on the scale of the transmission; hence, it will affect the type of application supported by the corresponding localization technique. So, it is important to notice vitality of localization in current and future wireless systems in any and all applications and scenarios.

\begin{tabular}{|l|l|l|l|}
\hline $\begin{array}{l}\text { Table 1 Comparisons of positioning techniques } \\
\text { Systems }\end{array}$ & $\begin{array}{l}\text { Localization } \\
\text { Technique }\end{array}$ & Range & Accuracy \\
\hline Dolphin [143][144] & ToA, trilateration & Room & $2 \mathrm{~cm}$ \\
\hline RFID/INS [145]-[150] & RSS/INS & Indoor & $2 \mathrm{~m}$ \\
\hline UWB [151]-[154] & $\begin{array}{l}\text { TDoA/ToA, } \\
\text { trilateration }\end{array}$ & $15 \mathrm{~m}$ & $10 \mathrm{~cm}$ \\
\hline RFID/FPM [155] & RSS/INS & Indoor & $1.7 \mathrm{~m}$ \\
\hline Land Marc [156][157] & RSS, triangulation & $50 \mathrm{~m}$ & $1-2 \mathrm{~m}$ \\
\hline GPS [158][159] & ToA, trilateration & Global & $1-5 \mathrm{~m}$ \\
\hline Radar [160][161] & RSS, triangulation & $\begin{array}{l}\text { Room } \\
\text { scale }\end{array}$ & $2-3 \mathrm{~m}$ \\
\hline Cricket [162] & $\begin{array}{l}\text { ToA, trilateration } \\
\text { FSSI theoretical } \\
\text { propagation model }\end{array}$ & $10 \mathrm{~m}$ & $2 \mathrm{~cm}$ \\
\hline Active Bats [163] & ToA, trilateration & $50 \mathrm{~m}$ & $9 \mathrm{~cm}$ \\
\hline Active Badge [164][165] & ToA, trilateration & $5 \mathrm{~m}$ & $7 \mathrm{~cm}$ \\
\hline COMPASS [166][167] & RSS, triangulation & $15 \mathrm{~m}$ & $1.65 \mathrm{~m}$ \\
\hline WhereNet [168][169] & RSS, triangulation & $20 \mathrm{~m}$ & $2-3 \mathrm{~m}$ \\
\hline LiFS [79] & $\begin{array}{l}\text { Fingerprinting } \\
\text { Database }\end{array}$ & $9 \mathrm{~m}$ \\
\hline Bluetooth [170][171] & $\begin{array}{l}\text { Indoor } \\
\text { FSI }\end{array}$ & $2-5 \mathrm{~m}$ \\
\hline
\end{tabular}

\section{PERSPECTIVES AND CHALLENGES}

In this section, we summarize the perspectives and challenges in indoor localization. We should mention that many challenges and pitfalls have been addressed in the previous sections. The target of this section is just to summarize on the main ones and 
provide some details on those which have not been addressed so far.

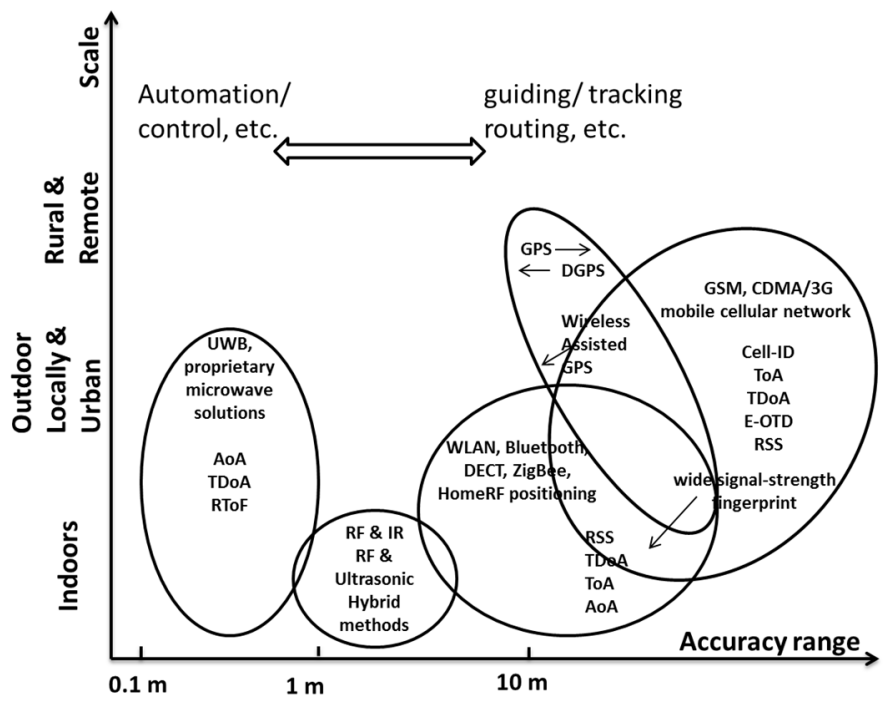

Figure 11- List of various wireless-based positioning systems

A. Constructing radio fingerprinting map with less human participation

Although the fingerprinting-based approach provides satisfactory accuracy, one often has to reconstruct or update the fingerprinting map to capture the features of a new environment (for example, when new sensors are installed), which is extremely time-consuming. Moreover, the complexity of the fingerprinting-based approaches is proportional to the number of entitles one wants to locate. For example, in order to locate a laptop and a smart phone, we need to build up a fingerprint map for each of them due to the heterogeneity of these devices. So the main challenge to address here is to have a unified framework for radio fingerprinting maps independently of entitles.

\section{B. Combining various non-radio techniques}

The use of non-radio technologies (IMU, visual sensors) can compensate for the errors from the existing wireless positioning technology. The improved accuracy is achieved by the additional installations of the costly equipment; therefore investigating a cost-effective wireless positioning system is still a promising direction.

\section{Integrating various wireless positioning solutions.}

A variety of wireless sensors can be used for positioning services. Different sensors are working with different physical principles. Combining the measurements from different positioning systems can improve performance of the overall solution.

\section{Security and Privacy}

The security and privacy factors are considered important to discuss with Indoor Positioning Systems (IPSs) within Personal Network [12], where users position people and objects in their home. The user cares if he is being tracked and his activities are being known by someone. Typical IPSs have threats in WLANIPS environment. For instance, positioning system based on RSS measurements threaten security since the mobile device collects the measurements of all AP devices and personal data such as AP ID is sent to the IPS server [172]. Additionally, the privacy in IPSs can be enhanced via a controlled access to the information distribution and location information. As well, security and privacy can be improved in IPSs from the software and system architecture perspectives. For instance, position system architecture dealing with self-localization can guarantee high degree of privacy and security for users when the estimation of the position is done at the target device [12]. Hence, the location information can't be accessed by anyone in Personal Networks (PNs) if the target device doesn't provide it to an entity.

\section{E. Scalability}

A scalable positioning system means that it functions properly when its scope gets larger. Usually, the performance of localization reduces with the increase in the distance between the transmitter and receiver. Further, a positioning system may require scaling on 2 axes, density and geography. Geographic scaling represents the coverage of an area or volume, whereas density scaling represents the number of units positioned per unit geographic space or area per time period. Wireless signal channels may turn out to be congested as more area is covered or the units in such area are crowded; hence, further computation or communication infrastructure may be required to do localization. In addition, the dimension of a system is another metric for scalability. A positioning system may locate objects in 2-D space, 3-D space or in both. So far, most of the proposed techniques are dealing with $2 \mathrm{D}$ while the recent recommendations from different standardization committees require 3D information with high accuracy (FCC recommendation for instance). This is then another important aspect to be tackled in the future.

\section{F. Complexity}

Positioning systems have complexity in terms of software, hardware, and operation factors. Specifically, software complexity represents the complexity of computations in a positioning algorithm. If a centralized server side handles the computations, location estimation is performed quickly because of the existence of adequate power supply and great processing capability. However, if the computations are performed at the mobile user, complexity becomes evident since mobile users have weak processing power and short battery life; thus, positioning techniques with low complexity are preferred in this case. Additionally, representing complexity of various positioning techniques through a formula analytically is really a difficult task; hence, the time spent for computations should be considered. Also, location rate is used as an indicator for complexity. Oppositely, we have location lag that represents the delay between movement of a mobile user to a new position and the reporting of this new position by the system. 


\section{G. Accuracy vs. Cost-effective.}

Different levels of accuracy are achieved with different positioning systems. For instance, an accuracy of $2 \mathrm{~m}$ to $3 \mathrm{~m}$ is achieved with RADAR [160]. On the other hand, $2 \mathrm{~cm}$ accuracy is achieved with a cricket system that is based on ultrasonic signals [162]. Furthermore, WLAN-based positioning system utilizes the existing WLAN infrastructures for localization; therefore, the positioning accuracy highly depends on the environments and placements of the wireless sensor nodes.

Other positioning systems usually require previous installation of the beacons in the environment. Therefore, in practice, it is very challenging to meet accuracy requirement and at the same time keep within minimum number of beacons and mobile device costs. The trade-off between accuracy and cost consists of a capital challenge in indoor scenario since from one side; the accuracy suffers from the existence of obstacles and multipath transmission while the cost is quite affordable due to the availability of signals (such as WLAN) at reduced cost.

\section{H. Applications and Accuracy}

The accuracy of the localization techniques highly depends on the applications. While some techniques are working perfectly in indoor scenarios, for instance, they might not be applicable in medical applications such as in Wireless Body Area Networks due to the precision requirement (in $\mathrm{mm}$ ). This is a general challenge which should be tackled in any application and environment.

Particularly, in mmWave, a promising candidate for 5G networks, the accuracy and means of localization are still open research issues to be solved. Nevertheless, the importance of high accuracy is primordial in this environment.

\section{REFERENCES}

[1] A. K"upper. Location-based services. Wiley, 2005

[2] F. L. Piccolo, "A new cooperative localization method for UDS cellular networks", in Proc. IEEE Global Telecommun. Conf., Dec. 2008.

[3] C. Mensing, S. Sand, and A. Dammann, "Hybrid data fusion and tracking for positioning with GNSS and 3GPP-LTE," International Journal of Navigation and Observation, vol. 2010, Aug. 2010.

[4] C. Mensing, S. Sand, A. Dammann, and W. Utschick, "Interferenceaware location estimation in cellular OFDM communications systems", in Proc. IEEE Int. Conf. Commun., Jun. 2009.

[5] C. Mensing, S. Sand, A. Dammann and W. Utschick, "DataAided Location Estimation in Cellular OFDM Communications Systems," Global Telecommunications Conference, 2009. GLOBECOM 2009. IEEE, Honolulu, HI, 2009, pp. 1-7.

[6] A. H. Sayed, A. Tarighat, and N. Khajehnouri, "Network-based wireless location", IEEE Signal Process. Mag., vol. 22, no. 4, pp. 12-23, Jul. 2005.

[7] Ziming He; Yi Ma; Tafazolli, R.; , "A hybrid data fusion based cooperative localization approach for cellular networks," Wireless Communications and Mobile Computing Conference (IWCMC), 2011 7th International, pp.162-166, 4-8 July 2011, doi: 10.1109/IWCMC.2011.5982409
[8] Ijaz, Faheem, et al. "Indoor positioning: A review of indoor ultrasonic positioning systems." Advanced Communication Technology (ICACT), 2013 15th International Conference on. IEEE, 2013.

[9] Koyuncu, Hakan, and Shuang Hua Yang. "A survey of indoor positioning and object locating systems." IJCSNS International Journal of Computer Science and Network Security 10.5 (2010): 121-128.

[10] Zahid Farid, Rosdiadee Nordin, and Mahamod Ismail, "Recent Advances in Wireless Indoor Localization Techniques and System," Journal of Computer Networks and Communications, vol. 2013, Article ID 185138, 12 pages, 2013. doi:10.1155/2013/185138

[11] H. Liu, H. Darabi, P. Banerjee, and J. Liu. Survey of Wireless Indoor Positioning Techniques and Systems. IEEE Transactions on Systems, Man and Cybernetics, 37(6):10671080, 2007.

[12] Y. Gu, A. Lo, and I. Niemegeers, "A survey of indoor positioning systems for wireless personal networks", IEEE Comm. Surveys and Tutorials, vol. 11, no. 1, pp. 13-32, First Quarter 2009.

[13] Disha A. "A Comparative Analysis on indoor positioning Techniques and Systems". International Journal of Engineering Research and Applications. 2013;3:1790-6

[14] Al-Ammar, M.A.; Alhadhrami, S.; Al-Salman, A.; Alarifi, A.; Al-Khalifa, H.S.; Alnafessah, A.; Alsaleh, M., "Comparative Survey of Indoor Positioning Technologies, Techniques, and Algorithms," in Cyberworlds (CW), 2014 International Conference on , vol., no., pp.245-252, 6-8 Oct. 2014

[15] Vo Q. Duy and De P., "A Survey of Fingerprint-Based Outdoor Localization," in IEEE Communications Surveys \& Tutorials, vol. 18, no. 1, pp. 491-506, Firstquarter 2016.

[16] Montorsi, F.; Mazuelas, S.; Vitetta, G.M.; Win, M.Z., "On the Performance Limits of Map-Aware Localization," Information Theory, IEEE Transactions on , vol.59, no.8, pp.5023,5038, Aug. 2013

[17] Chandrasekaran, G.; Ergin, M.A.; Jie Yang; Song Liu; Yingying Chen; Gruteser, M.; Martin, R.P., "Empirical Evaluation of the Limits on Localization Using Signal Strength," Sensor, Mesh and Ad Hoc Communications and Networks, 2009. SECON '09. 6th Annual IEEE Communications Society Conference on , pp.1,9, 22-26 June 2009

[18] Hongbo Liu; Jie Yang; Sidhom, S.; Yan Wang; Yingying Chen; Fan Ye, "Accurate WLAN Based Localization for Smartphones Using Peer Assistance," Mobile Computing, IEEE Transactions on , vol.13, no.10, pp.2199,2214, Oct. 2014

[19] Guvenc, Ismail, Sinan Gezici, and Zafer Sahinoglu. "Fundamental limits and improved algorithms for linear leastsquares wireless position estimation." Wireless Communications and Mobile Computing 12.12 (2012): 10371052

[20] D. Dardari, A. Conti, J. Lien and M.Z. Win, "The Effect of Cooperation on UWB-Based Positioning Systems Using Experimental Data", EURASIP Journal on Advances in Signal Processing, Special Issue on Wireless Cooperative Networks, Vol. 2008, pp.191-195, 2008 
[21] A.Conti, D. Dardari, and M. Z. Win, "Experimental Results on Cooperative UWB Based Positioning Systems", in Proc. IEEE ICUWB'08, vol. 1, pp. 191-195, Hannover, Sept. 2008

[22] A. Yassine, Y. Nasser, M. Awad, B. Uguen, "Hybrid positioning data fusion in heterogeneous networks with critical hearability", EURASIP Journal on Advances in Signal Processing, December 2014, pages:16, 2014:215 doi:10.1186/1687-1499-2014-215

[23] S. Gezici, Z. Tian, G. Giannakis, H. Kobayashi, A. Molisch, H. Poor, and Z. Sahinoglu, "Localization via ultra-wideband radios: a look at positioning aspects for future sensor networks," IEEE Signal Processing Magazine, vol. 22, no. 4, pp. 70-84, July 2005.

[24] W. Mekonnen, E. Slottke, H. Luecken, C. Steiner, and A. Wittneben, "Constrained Maximum Likelihood Positioning for UWB Based Human Motion Tracking," International Conference on Indoor Positioning and Indoor Navigation, IPIN 2010, Zurich, Switzerland, pp. 1-10, Sept. 2010.

[25] Z. Sahinoglu, S. Gezici, and I. Guvenc. Ultra-wideband Positioning Systems. Cambridge, 2008.

[26] Alarifi, Abdulrahman et al. "Ultra Wideband Indoor Positioning Technologies: Analysis and Recent Advances ." Ed. Lyudmila Mihaylova, Byung-Gyu Kim, and Debi Prosad Dogra. Sensors (Basel, Switzerland) 16.5 (2016): 707. PMC. Web. 19 Aug. 2016.

[27] You Zheng; Baala, O.; Caminada, A., "A new approach to design a WLAN-based positioning system," in Signal Processing and Communication Systems (ICSPCS), 2010 4th International Conference on , vol., no., pp.1-8, 13-15 Dec. 2010. doi: 10.1109/ICSPCS.2010.5709652

[28] Long Vu-Hoang; Hung Nguyen-Manh; Chinh Phan-Duy; Dung Le-Quang; Thanh Do-Van; Vinh Tran-Quang, "A new technique to enhance accuracy of WLAN fingerprinting based indoor positioning system," in Communications and Electronics (ICCE), 2014 IEEE Fifth International Conference on , vol., no., pp.270-275, July 30 2014-Aug. 1 2014. doi: 10.1109/CCE.2014.6916714

[29] R. Zhang, F. Hoeflinger, O. Gorgis, and L.M. Reindl, "Indoor localization using inertial sensors and ultrasonic rangefinder," in Proc. Int. Conf. on Wireless Communications and Signal Processing (WCSP 2011), Nanjing, China, Nov. 2011, pp. 1-5.

[30] A.R. Jimenez, F. Seco, J.C. Prieto, and J. Guevara, "Indoor Pedestrian navigation using an INS/EKF framework for yaw drift reduction and a foot-mounted IMU," in Proc. 7th Workshop on Positioning, Navigation and Communication (WPNC 2010), Dresden, Germany, Mar. 2010, pp. 135-143.

[31] Hwan-Joo Kwak; Dong-Hun Lee; Jung-Moon Hwang; JungHan Kim; Chong-Kap Kim; Gwi-Tae Park, "Improvement of the inertial sensor-based localization for mobile robots using multiple estimation windows filter," in Intelligent Robots and Systems (IROS), 2012 IEEE/RSJ International Conference on, vol., no., pp.876-881, 7-12 Oct. 2012. doi: 10.1109/IROS.2012.6386032

[32] Lei Fang, Wenliang Du and Peng Ning, "A beacon-less location discovery scheme for wireless sensor networks," Proceedings IEEE 24th Annual Joint Conference of the IEEE Computer and Communications Societies., 2005, pp. 161-171 vol. 1.

[33] Nazir, U.; Arshad, M.A.; Shahid, N.; Raza, S.H., "Classification of localization algorithms for wireless sensor network: A survey," in Open Source Systems and Technologies (ICOSST), 2012 International Conference on, vol., no., pp.15, 20-22 Dec. 2012

[34] Y.-C. Lee, Christiand, W. Yu, and J.-I. Cho, "Adaptive localization for mobile robots in urban environments using low-cost sensors and enhanced topological map," in Proc. IEEE 15th International Conference on Advanced Robotics (ICAR 2011), Tallinn, Estonia, Jun. 2011, pp. 569-575.

[35] D.J. Kim, M.K. Kim, K.S. Lee, H.G. Park, and M.H. Lee, "Localization system of autonomous vehicle via Kalman filtering," in Proc. Int. Conf. on Control, Automation and Systems, Gyeonggi-do, Korea, Oct. 2011, pp. 934-937.

[36] Le, T.N.; Chong, P.H.J.; Xue Jun Li; Wai Yie Leong, "A Simple Grid-Based Localization Technique in Wireless Sensor Networks for Forest Fire Detection," in Communication Software and Networks, 2010. ICCSN '10. Second International Conference on , vol., no., pp.93-98, 26-28 Feb. 2010. doi: 10.1109/ICCSN.2010.108

[37] D. Serant, P. Thevenon, M.-L. Boucheret, O. Julien, C. Macabiau, S. Corazza, M. Dervin, and L. Ries, "Development and validation of an OFDM/DVB-T sensor for positioning," in Position Location and Navigation Symposium (PLANS), 2010 IEEE/ION, May 2010.

[38] F. Alizadeh-shabdiz and K. Pahlavan, "Estimation of position using WLAN access point radio propagation characteristics in a WLAN positioning system," Patent 7515 578, Apr., 2009. Available: http://www.freepatentsonline.com/7515578.html

[39] C. Yang, T. Nguyen, D. Venable, M. White, and R. Siegel, "Cooperative position location with signals of opportunity," in Proceedings of the IEEE 2009 National Aerospace Electronics Conference (NAECON), Jul. 2009.

[40] Enright, M.A.; Kurby, C.N., "A signals of opportunity based cooperative navigation network," Aerospace \& Electronics Conference (NAECON), Proceedings of the IEEE 2009 National, vol., no., pp.213,218, 21-23 July 2009

[41] Coluccia, A.; Ricciato, F.; Ricci, G., "Positioning Based on Signals of Opportunity," Communications Letters, IEEE , vol.18, no.2, pp.356,359, February 2014

[42] Chun Yang; Thao Nguyen; Blasch, E., "Mobile positioning via fusion of mixed signals of opportunity," Aerospace and Electronic Systems Magazine, IEEE , vol.29, no.4, pp.34,46, April 2014

[43] Robinson, M.; Ghrist, R., "Topological Localization Via Signals of Opportunity," Signal Processing, IEEE Transactions on, vol.60, no.5, pp.2362,2373, May 2012

[44] A. De Angelis, M. Dionigi, R. Moschitta, A. Giglietti, and P. Carbone, "Characterization and Modeling of an Experimental UWB Pulse-Based Distance Measurement System," IEEE Transactions on Instrumentation and Measurement, vol. 58, no. 5, pp. 1479 - 1486, May 2009.

[45] H. Wymeersch, J. Lien, and M. Win, "Cooperative 1ocalization in wireless networks," Proceedings of the IEEE, vol. 97, no. 2, pp. 427-450, Feb. 2009.

[46] Kietlinski-Zaleski, J.; Yamazato, T.; Katayama, M., "Experimental validation of ToA UWB positioning with two receivers using known indoor features," Position Location and Navigation Symposium (PLANS), 2010 IEEE/ION , vol., no., pp.505,509, 4-6 May 2010 
[47] XU Benchong, CHEN Junjie. Localization Algorithms for Wireless Sensor Networks Based on Feasibility of Anchor Nodes Disposing, Measurement \& Control Techniques,2007,8, P:46-51

[48] Wang Jianguo; Wang Zhongsheng; Zhang Ling; Shi Fei; Song Guohua, "A New Anchor-Based Localization Algorithm for Wireless Sensor Network," in Distributed Computing and Applications to Business, Engineering and Science (DCABES), 2011 Tenth International Symposium on, vol., no., pp.239-243, 14-17 Oct. 2011. doi: 10.1109/DCABES.2011.5

[49] A. P. Jardosh, K. Papagiannaki, E. M. Belding, K. C. Almeroth, G. Iannaccone, and B. Vinnakota, "Green WLANs: on-demand WLAN infrastructures," Mobile Networks and Applications, vol. 14, no. 6, pp. 798-814, Dec. 2009.

[50] K. Chintalapudi, A. P. Iyer, and V. N. Padmanabhan, "Indoor localization without the pain," in Proc. of Mobile Computing and Networking (MobiCom), 2010, pp. 173-184.

[51] J. Paek, J. Kim, and R. Govindan, "Energy-Efficient RateAdaptive GPSbased Positioning for Smartphones," in Proc. of MobiSys, 2010.

[52] Dhondge, K.; Hyungbae Park; Baek-Young Choi; Sejun Song, "Energy-Efficient Cooperative Opportunistic Positioning for Heterogeneous Mobile Devices," Computer Communications and Networks (ICCCN), 2012 21st International Conference on , vol., no., pp.1,6, July 30 2012-Aug. 2012

[53] Zheng Sun; Farley, R.; Kaleas, T.; Ellis, J.; Chikkappa, K., "Cortina: Collaborative context-aware indoor positioning employing RSS and RToF techniques," Pervasive Computing and Communications Workshops (PERCOM Workshops), 2011 IEEE International Conference on , vol., no., pp.340,343, 21-25 March 2011

[54] Yubin Xu; Mu Zhou; Lin Ma; Weixiao Meng, "3GPE: An energy efficient probabilistic fingerprint-assisted localization in indoor WLAN areas," Communications (ICC), 2012 IEEE International Conference on , vol., no., pp.32,36, 10-15 June 2012

[55] Lemelson, H., Kjærgaard M. B., Hansen R., and King T. "Error estimation for indoor 802.11 location fingerprinting." in Proc. of the Fourth Int.Symposium on Location and Context Awareness (LoCA 2009), May 2009.

[56] Lemelson, H.; Kopf, S.; King, T.; Effelsberg, W., "Improvements for 802.11-Based Location Fingerprinting Systems," in Computer Software and Applications Conference, 2009. COMPSAC '09. 33rd Annual IEEE International, vol.1, no., pp.21-28, 20-24 July 2009

[57] http://www.ict-where2.eu/.

[58] C. Pedersen, T. Pedersen, and B. H. Fleury. A variational message passing algorithm for sensor self-localization in wireless networks. In Proc. IEEE ISIT 2011, pages 2158-2162, Saint-Petersburg, Russia, August 2011.

[59] V. Savic, H. Wymeersch, F. Penna, and S. Zazo. Optimized edge appearance probability for cooperative localization based on tree-reweighted nonparametric belief propagation. In Proc. IEEE ICASSP 2011, pages 3028-3031, Prague, Czech Republic, May 2011.

[60] H. Noureddine, N. Gresset, D. Castelain, and R. Pyndiah. A New Variant of Nonparametric Belief Propagation for SelfLocalization. In Proc. IEEE ICT 2010, pages 822-827, Doha, Qatar, April 2010.
[61] S. Hadzic, J. Bastos, and J. Rodriguez. Reference node selection for cooperative positioning using coalition formation games. In Proc. WPNC 2012, pages 105-108, Dresden, Germany, March 2012.

[62] S. Zirari and B. Denis. Comparison of links selection criteria for mobile terminal positioning in cooperative heterogeneous networks. In Proc. SoftCOM 2012, Split, Croatia, September 2012.

[63] A. Conti, M. Guerra, D. Dardari, N. Decarli, and M.Z. Win. Network Experimentation for Cooperative Localization. IEEE JSAC, 30(2):467- 475, February 2012.

[64] Denis, B., et al. "Cooperative and heterogeneous indoor localization experiments." Communications Workshops (ICC), 2013 IEEE International Conference on. IEEE, 2013.

[65] Sand, S.; Mensing, C.; Ma, Y.; Tafazolli, R.; Yin, X.; Figueiras, J.; Nielsen, J.; Fleury, B.H., "Hybrid Data Fusion and Cooperative Schemes for Wireless Positioning," Vehicular Technology Conference, 2008. VTC 2008-Fall. IEEE 68th , vol., no., pp.1,5, 21-24 Sept. 2008

[66] Kemppi, P.; Rautiainen, T.; Ranki, V.; Belloni, F.; Pajunen, J., "Hybrid positioning system combining angle-based localization, pedestrian dead reckoning and map filtering," Indoor Positioning and Indoor Navigation (IPIN), 2010 International Conference on , vol., no., pp.1,7, 15-17 Sept. 2010

[67] Tarrío, Paula, Juan A. Besada, and José R. Casar. "Fusion of RSS and inertial measurements for calibration-free indoor pedestrian tracking." Information Fusion (FUSION), 2013 16th International Conference on. IEEE, 2013.

[68] H. Wang, H. Lenz, A. Szabo, J. Bamberger and U. Hanebeck, "WLANBased Pedestrian Tracking Using Particle Filters and Low-Cost MEMS Sensors", Proc. of the 4th Workshop on Positioning, Navigation and Communication, pp. 1-7, 2007.

[69] O. Woodman and R. Harle, "Pedestrian localisation for indoor environments", Proc. of the 10th Int. Conf. on Ubiquitous Computing, pp. 114-123, 2008.

[70] B. Denis, M. Maman, and L Ouvry, "On the Scheduling of Ranging and Distributed Positioning Updates in Cooperative IR-UWB Networks," in Proceedings of IEEE International Conference on Ultra-Wideband (ICUWB), pp. 370 -375, Sep. 2009.

[71] D. Lieckfeldt, J. You, and D. Timmermann, "An algorithm for distributed beacon selection," in IEEE International Conference on Pervasive Computing and Communications, Hong Kong, China, pp. 318-323, March 2008.

[72] K. Das and H. Wymeersch, "Censored cooperative positioning foe dense wireless networks," in IEEE 21st International Symposium on Personal, Indoor and Mobile Radio Communications Workshops (PIMRC Workshops), pp.262266, 26-30 September 2011.

[73] J. Liu and Y. Zhang, "Error control in distributed node selflocalization," in EURASIP Journal on Advances in Signal Processing, January 2008.

[74] Savic, V.; Zazo, S., "Reducing Communication Overhead for Cooperative Localization Using Nonparametric Belief Propagation," in Wireless Communications Letters, IEEE , vol.1, no.4, pp.308-311, August 2012

[75] Hadzic, S.; Rodriguez, J., "Utility based node selection scheme for cooperative localization," Indoor Positioning and Indoor 
Navigation (IPIN), 2011 International Conference on , vol., no., pp.1,6, 21-23 Sept. 2011

[76] M. B. Kjærgaard, "A taxonomy for radio location fingerprinting," in Location-and Context-Awareness. Springer Berlin Heidelberg, 2007, pp. 139-156.

[77] K. El-Kafrawy, M. Youssef, A. El-Keyi, and A. Naguib, "Propagation modeling for accurate indoor wlan rss-based localization," in Proc. Of the IEEE 72nd Vehicular Technology Conference Fall (VTC 2010-Fall), Ottawa, Canada, 6-9, September 2010, pp. 1-5.

[78] T. Deyle, C. Kemp, and M. Reynolds, "Probabilistic UHF RFID tag pose estimation with multiple antennas and a multipath RF propagation model," in Proc. of the 2008 IEEE/RSJ Int. Conf. on Intelligent Robots and Systems (IROS 2008), Nice, France, September 2008, pp. 1379-1384.

[79] Z. Yang, C. Wu, and Y. Liu, "Locating in fingerprint space: Wireless indoor localization with little human intervention," in Proceedings of the 18th Annual International Conference on Mobile Computing and Networking, ser. Mobicom '12, 2012, pp. 269-280.

[80] A. Rai, K. K. Chintalapudi, V. N. Padmanabhan, and R. Sen, "Zee: Zeroeffort crowdsourcing for indoor localization," in Proceedings of the 18th Annual International Conference on Mobile Computing and Networking, ser. Mobicom '12, 2012, pp. 293-304.

[81] M. Raspopoulos, C. Laoudias, L. Kanaris, A. Kokkinis, C. Panayiotou, and S. Stavrou, "3d ray tracing for deviceindependent fingerprint-based positioning in wlans," in 2012 9th Workshop on Positioning Navigation and Communication (WPNC 2012), March 2012, pp. 109-113.

[82] Jochen Seitz, Lucila Patino-Studencka, Bernd Schindler, Stephan Haimerl, Javier Gutierrez Boronat, Steffen Meyer, Jörn Thielecke. "Sensor Data Fusion for Pedestrian Navigation Using WLAN and INS," In Symposium Gyro Technology 2007, Karlsruhe, 2007.

[83] M. Jin, B. Koo, S. Lee, C. Park, M. Lee and S. Kim, "IMUAssisted Nearest Neighbor Selection for Real-Time WLAN Fingerprinting Positioning," in Proc. IEEE International Conference on Indoor Positioning and Indoor Navigation (IPIN), Busan, Korea, Oct. 2014.

[84] W. Xiao, W. Ni, and Y. K. Toh, "Integrated WLAN Fingerprinting and Inertial Sensing for Indoor Positioning," International conference on Indoor Positioning and Indoor Navigation. Guimaraes, Portugal, September 2011.

[85] Antonio J. Ruiz-Ruiz, Pedro E. Lopez-de-Teruel, Oscar Canovas. "A multisensor LBS using SIFT-based 3D models," 2012 International Conference on Indoor Positioning and Indoor Navigation, November 13-15, 2012.

[86] K. Hattori, R. Kimura, N. Nakajima, T. Fujii, Y. Kado, B. Zhang, T. Hazugawa, K. Takadama, "Hybrid Indoor Location Estimation System Using Image Processing and WLAN Strength," in the 9th International conference on Wireless network and Information System, Shanghai China, Dec 2009:406-411.

[87] T. Germa, F. Lerasle, N. Ouadah, V. Cadenat, "Vision and RFID data fusion for tracking people in crowds by a mobile robot," Computer Vision and Image Understanding, Volume 114, Issue 6, June 2010, Pages 641-651.

[88] Dirk Schulz, Dieter Fox, Jeffrey Hightower. "People Tracking with Anonymous and ID-Sensors Using Rao-Blackwellised
Particle Filters,” Proc. of the International Joint Conference on Artificial Intelligence (IJCAI), 2003

[89] Muhammad Irshan Khan and Jari Syrjarinne. "Investigating Effective Methods for Integration of Building's Map with Low cost Inertial Sensors and WLAN-based Positioning," 2013 International Conference on Indoor Positioning and Indoor Navigation, 28th-31th, October 2013.

[90] Leung, K.Y.K.; Barfoot, T.D.; Liu, H.H.T., "Distributed and decentralized cooperative simultaneous localization and mapping for dynamic and sparse robot networks," Robotics and Automation (ICRA), 2011 IEEE International Conference on, vol., no., pp.3841,3847, 9-13 May 2011.

[91] Heon-Cheol Lee; Seung-Hee Lee; Seung-Hwan Lee; Tae-Seok Lee; Doo-Jin Kim; Kyung-Sik Park; Kong-Woo Lee; BeomHee Lee, "Comparison and analysis of scan matching techniques for Cooperative-SLAM," Ubiquitous Robots and Ambient Intelligence (URAI), 2011 8th International Conference on , vol., no., pp.165,168, 23-26 Nov. 2011

[92] Ali, A.M.; Nordin, Md Jan, "SIFT based monocular SLAM with multi-clouds features for indoor navigation," TENCON 2010 - 2010 IEEE Region 10 Conference, vol., no., pp.2326,2331, 21-24 Nov. 2010

[93] Jiun-Fu Chen; Chieh-Chih Wang, "Simultaneous localization and mapping using a short-range passive RFID reader with sparse tags in large environments," Advanced Robotics and its Social Impacts (ARSO), 2010 IEEE Workshop on , vol., no., pp.136,141, 26-28 Oct. 2010

[94] Kuen-Han Lin; Chieh-Chih Wang, "Stereo-based simultaneous localization, mapping and moving object tracking," Intelligent Robots and Systems (IROS), 2010 IEEE/RSJ International Conference on , vol., no., pp.3975,3980, 18-22 Oct. 2010

[95] Kosmatopoulos, E.B.; Rovas, D.V.; Doitsidis, L.; Aboudolas, K.; Roumeliotis, S.I., "A generic framework for scalable and convergent multi-robot active simultaneous localization, mapping and target tracking," Control \& Automation (MED), 2011 19th Mediterranean Conference on , vol., no., pp.151,156, 20-23 June 2011

[96] Zhao Xinzhe; Zhang Simin, "Distributed strong tracking unscented particle filter for simultaneous localization and mapping," Control Conference (CCC), 2014 33rd Chinese , vol., no., pp.978,983, 28-30 July 2014

[97] Brian Ferris, Dieter Fox, and Neil Lawrence. "WLAN-SLAM using Gaussian process latent variable models," In Proceedings of the 20th international joint conference on Artifical intelligence (IJCAI'07), Rajeev Sangal, Harish Mehta, and R. K. Bagga (Eds.). Morgan Kaufmann Publishers Inc., San Francisco, CA, USA, 2480-2485.

[98] Joseph Huang, David Millman, Morgan Quigley, David Stavens, Sebastian Thrun and Alok Aggarwal. "Efficient, generalized indoor WLAN GraphSLAM," in 2011 IEEE International Conference on Conference: Robotics and Automation (ICRA), May 2011, Shanghai.

[99] Piotr Mirowski, Tin Kam Ho, Saehoon Yi, Michael MacDonald. "SignalSLAM: Simultaneous localization and mapping with mixed WLAN, Bluetooth, LTE and magnetic signals," in 2013 International Conference on Indoor Positioning and Indoor Navigation (IPIN).

[100] Z. Yang, C. Wu, and Y. Liu, "Locating in Fingerprint Space: Wireless Indoor Localization with Little Human Intervention," in Proc. of ACM MobiCom , 2012. 
[101] G. Shen, Z. Chen, P. Zhang, T. Moscibroda, and Y. Zhang, "Walkie- Markie: indoor pathway mapping made easy," in Proc. of USENIX NSDI, 2013.

[102] A. Rai, K. K. Chintalapudi, V. N. Padmanabhan, and R. Sen, "Zee: Zero-effort Crowdsourcing for Indoor Localization," in Proc. of ACM MobiCom, 2012

[103] J. Yin, Q. Yang, and L. M. Ni, "Learning adaptive temporal radio maps for signal-strength-based location estimation," Mobile Computing, IEEE Transactions on, vol. 7, no. 7, pp. 869-883, 2008.

[104] Z. Sun, Y. Chen, J. Qi, and J. Liu, "Adaptive localization through transfer learning in indoor wi-fi environment," in Proc. of IEEE ICMLA, 2008.

[105] H. Wang, S. Sen, A. Elgohary, M. Farid, M. Youssef, and R. R. Choudhury, "No Need to War-drive: Unsupervised Indoor Localization," in Proc. of ACM MobiSys, 2012.

[106] L. Zhang, K. Liu, Y. Jiang, X.-Y. Li, Y. Liu and P. Yang, "Montage: Combine frames with movement continuity for realtime multi-user tracking," in Proc. of IEEE INFOCOM, 2014

[107] Myerson R. B. (1997). Game theory, Analysis of conflict, Harvard University Press, ISBN: 0-674- 34115-5, Cambridge, MA, USA.

[108] V. Srivastava et al., "Using game theory to analyze wireless ad hoc networks," in IEEE Communications Surveys \& Tutorials, vol. 7, no. 4, pp. 46-56, Fourth Quarter 2005.

[109] Ghassemi F. \& Krishnamurthy V. (2008a). A Cooperative Game-Theoretic Measurement Allocation Algorithm for Localization in Unattended Ground Sensor Networks, Proceedings of 11th International Conference on Information Fusion, pp. 1-7, ISBN: 978-3-8007-3092-6, Cologne, Germany, June 2008.

[110] B. Bejar, P. Belanovic, and S. Zazo, "Cooperative localization in wireless sensor networks using coalitional game theory," in 18th European Signal Processing Conference EUSIPCO 2010, Aalborg, Denmark, August 2010.

[111] G. A. Shah and M. Bozyigit, "Exploiting Energy-aware Spatial Correlation in Wireless Sensor Networks", in Proc. of 2nd International Conference on Communication Systems Software and Middleware (COMSWARE 2007), Bangalore, India, pp. 1-6, January 2007.

[112] Franchi, A.; Oriolo, G.; Stegagno, P., "Probabilistic mutual localization in multi-agent systems from anonymous position measures," Decision and Control (CDC), 2010 49th IEEE Conference on , vol., no., pp.6534,6540, 15-17 Dec. 2010

[113] Katalenic, A.; Draganjac, I.; Mutka, A.; Bogdan, S., "Fast visual tracking and localization in multi-agent systems," Industrial Electronics and Applications, 2009. ICIEA 2009. 4th IEEE Conference on , vol., no., pp.1864,1870, 25-27 May 2009

[114] ABI Research, "Location Technology Research Service,” Oct 2013.

[115] "Consumer Applications for Indoor Positioning”, Loctronix The Insider, Vol. 1, No. 1, Available at: http://goo.gl/PYtgwT

[116] D. Macagnano, G. Destino and G. Abreu "Indoor Positioning: a Key Enabling Technology for IoT Applications," IEEE World Forum on Internet of Things (WF-IoT), p. 117, 2014.

[117] BUTLER FP7 project web page http://www.iotbutler.eu/about-butler
[118] "URUS:Ubiquitous networking Robots in Urban Settings," EU Project IST-1-045062, Web site: http://wwwiri.upc.es/groups/urus/.

[119] Aufrere, R.; Karam, N.; Chausse, F.; Chapuis, R., "A state exchange approach in real conditions for multi-robot cooperative localization," Intelligent Robots and Systems (IROS), 2010 IEEE/RSJ International Conference on , vol., no., pp.4346,4351, 18-22 Oct. 2010

[120] P. V. Fazenda and P. U. Lima, "Non-holonomic robot formations with obstacle compliant geometry," Conference Paper, September 2007, 6th IFAC Symposium on Intelligent Autonomous Vehicles.

[121] S. Mastellone, D. M. Stipanovic, C. R. Graunke, K. A. Intlekofer, and M. W. Spong, "Formation control and collision avoidance for multi-agent non-holonomic systems. theory and experiments," The International Journal of Robotics Research, vol. 27, pp. 107-126, January 2008.

[122] S. Loizou and K. Kyriakopoulos, "Navigation of multiple kinematically constrained robots," IEEE Transactions on Robotics, vol. 24, no. 1, pp. 221-231, Feb. 2008.

[123] Ayush, K.; Agarwal, N.K., "Real time visual SLAM using cloud computing," Computing, Communications and Networking Technologies (ICCCNT),2013 Fourth International Conference on , vol., no., pp.1,7, 4-6 July 2013

[124] Heon-Cheol Lee; Seung-Hwan Lee; Tae-Seok Lee; Doo-Jin Kim; Beom-Hee Lee, "A survey of map merging techniques for cooperative-SLAM," Ubiquitous Robots and Ambient Intelligence (URAI), 2012 9th International Conference on , vol., no., pp.285,287, 26-28 Nov. 2012

[125] M. Segura, H. Hashemi, C. Sisterna, and V. Mut. Experimental demonstration of self-localized Ultra-Wideband indoor mobile robot navigation system. In International Conference on Indoor Positioning and Indoor Navigation (IPIN), 2010. doi: 10.1109/IPIN.2010.5647457.

[126] Prorok, A.; Gonon, L.; Martinoli, A., "Online model estimation of ultra-wideband TDoA measurements for mobile robot localization," in Robotics and Automation (ICRA), 2012 IEEE International Conference on , vol., no., pp.807-814, 14-18 May 2012. doi: 10.1109/ICRA.2012.6224869

[127] Bai Yan; Lu Xiaochun, "Research on UWB indoor positioning based on TDoA technique," in Electronic Measurement \& Instruments, 2009. ICEMI '09. 9th International Conference on , vol., no., pp.1-167-1-170, 16-19 Aug. 2009. doi: 10.1109/ICEMI.2009.5274900

[128] I. Qudah, P. Leijdekkers, and V. Gay, "Using mobile phones to improve medication compliance and awareness for cardiac patients," in Proc. Int. Conf. Pervas. Technol. Related Assisted Environ., 2010, pp. 1-7.

[129] D. U. Khan, K. A. Siek, J. Meyers, L. M. Haverhals, S. Cali, and S. E. Ross, "Designing a personal health application for older adults to manage medications," in Proc. Int. Health Inf. Symp., 2010, pp. 849-858.

[130] Haibo Ye; Tao Gu; Xianping Tao; Jian Lu, "F-Loc: Floor localization via crowdsourcing," in Parallel and Distributed Systems (ICPADS), 2014 20th IEEE International Conference on , vol., no., pp.47-54, 16-19 Dec. 2014

[131] Communications Security, Reliability and Interoperability Council III WG3 E9-1-1 Location Accuracy, "Report Leveraging LBS and Emerging Location Technologies for 
Indoor Wireless E9-1-1”, March 14, 2013. Available online at: http://goo.gl/hwGLp7

[132] FCC, THIRD FURTHER NOTICE OF PROPOSED RULEMAKING, February 21, 2014.

[133] Del Re, E., Morosi, S., Jayousi, S. Sacchi, C., "Salicesatellite-assisted localization and communication systems for emergency services," Proc. of of the 2009 1st International Conference on Wireless Communication, Vehicular Technology, Information Theory and Aerospace and Electronic Systems Technology, IEEE Wireless VITAE conference 2009.

[134] Del Re E., Jayousi, S. et. al., "SALICE Project: SatelliteAssisted Localization and Communication Systems for Emergency Services," IEEE AES Magazine, Sept. 2013, pp. 415.

[135] Open Mobile Alliance Location Working Group web page: http://openmobilealliance.org/aboutoma/workprogram/location/

[136] 3GPP TS 23.271 "Functional stage 2 description of Location Services (LCS)." Available at http://goo.gl/0EZTQw

[137] M. Mahfouz, C. Zhang, B. Merkl, M. Kuhn, and A. Fathy, "Investigation of high accuracy indoor 3-D positioning using UWB technology," IEEE Trans. Microw. Theory Tech., vol. 56, no. 6, pp. 1316-1330, Jun. 2008.

[138] Di Taranto, R. ; Muppirisetty, L. ; Raulefs, R. (2014) "Location-aware Communications for 5G Networks". IEEE signal processing magazine, vol. 31(6), pp. 102-112.

[139] D. Slock, "Location aided wireless communications," in Proc. Int. Symp. on Communications Control and Signal Processing, 2012, pp. 1-6.

[140] A. Dammann, G. Agapiou, J. Bastos, L. Brunel, M. García, J. Guillet, Y. Ma, J. Ma, J. J. Nielsen, L. Ping, R. Raulefs, J. Rodriguez, D. Slock, D. Yang, and N. Yi, "WHERE2 location aided communications," in Proc. European Wireless Conf., Apr.2013, pp. 1-8

[141] R. C. Daniels and R. W. Heath, "Link adaptation with position/motion information in vehicle-to-vehicle networks," IEEE Trans. Wireless Commun., vol. 11, no. 2,pp. 505-509, 2012.

[142] R. Di Taranto and H. Wymeersch, "Simultaneous routing and power allocation using location information," in Proc. Asilomar Conf. Signals, Systems and Computers, 2013, pp. 1700-1704.

[143] Mautz, R., "The challenges of indoor environments and specification on some alternative positioning systems," in Positioning, Navigation and Communication, 2009. WPNC 2009. 6th Workshop on , vol., no., pp.29-36, 19-19 March 2009. doi: 10.1109/WPNC.2009.4907800

[144] Halder, S. J., Park, J. G., \& Kim, W. (2011). Adaptive filtering for indoor localization using Zigbee RSSI and LQI measurement.

[145] Zhu M; Novel Positioning Algorithms for RFID-Assisted 2D MEMS INS Systems, 2008, Proceedings of the Institute of Navigation GNSS 2008 conference, Savannah, Georgia, US

[146] Retscher, G.; Qing Fu, "Continuous indoor navigation with RFID and INS," in Position Location and Navigation Symposium (PLANS), 2010 IEEE/ION , vol., no., pp.102-112, 4-6 May 2010. doi: 10.1109/PLANS.2010.5507242
[147] Ruiz, A.R.J.; Granja, F.S.; Honorato, J.C.P.; Rosas, J.I.G., "Pedestrian indoor navigation by aiding a foot-mounted IMU with RFID Signal Strength measurements," in Indoor Positioning and Indoor Navigation (IPIN), 2010 International Conference on , vol., no., pp.1-7, 15-17 Sept. 2010. doi: 10.1109/IPIN.2010.5646885

[148] Fu Q, Retscher G. Using RFID and INS for indoor positioning. 2009.

[149] Retscher, G., and Q. Fu. "An intelligent personal navigator integrating GNSS, RFID and INS for continuous position determination." Boletim de Ciências Geodésicas 15.5 (2009).

[150] Zhu, M., Retscher, G., \& Zhang, K. (2011). Integrated algorithms for RFID-based multi-sensor indoor/outdoor positioning solutions. Archiwum Fotogrametrii, Kartografii $i$ Teledetekcji, 22.

[151] D.Harmer; Ultra wide-band indoor positioning, Dec. 2004, online March 2008,http://esamultimedia.esa. int/docs/NavigationProjects

[152] Zheng Li; Dehaene, W.; Gielen, G., "A 3-tier UWB-based indoor localization system for ultra-low-power sensor networks," in Wireless Communications, IEEE Transactions on , vol.8, no.6, pp.2813-2818, June 2009. doi: 10.1109/TWC.2009.080602

[153] Stelios MA, Nick AD, Effie UD, Dimitris KM, Thomopoulos SC. An indoor localization platform for ambient assisted living using UWB. In Proceedings of the 6th international conference on advances in mobile computing and multimedia 2008 Nov 24 (pp. 178-182).

[154] Guoping Zhang; Krishnan, S.; Chin, F.; Chi Chung Ko, "UWB Multicell Indoor Localization Experiment System with Adaptive TDoA Combination," in Vehicular Technology Conference, 2008. VTC 2008-Fall. IEEE 68th, vol., no., pp.15, 21-24 Sept. 2008. doi: 10.1109/VETECF.2008.139

[155] Retscher G,Fu Q; Using Active RFID for Positioning in Navigation Systems, Proceedings of the 4th International Symposium on Location Based Services and Telecartography,Hong Kong, 2007

[156] Lionel M.NI, Yunhao Liu, Iu Cho Lau, Abhishek P. Patil; LANDMARC: Indoor Location Sensing Using Active RFID;

[157] Bergemann, S.; Sieck, J., "Adopting the LANDMARC Positioning System for $2.4 \mathrm{GHz}$ Band," in Computer Modeling and Simulation (EMS), 2011 Fifth UKSim European Symposium on , vol., no., pp.400-405, 16-18 Nov. 2011. doi: 10.1109/EMS.2011.55

[158] S. N. Razavi and O. Moselhi, "GPS-less indoor construction location sensing," in Automation in Construction. vol. 28, 2012, pp. 128-136.

[159] Drawil, N.M.; Amar, H.M.; Basir, O.A., "GPS Localization Accuracy Classification: A Context-Based Approach," in Intelligent Transportation Systems, IEEE Transactions on, vol.14, no.1, pp.262-273, March 2013. doi: 10.1109/TITS.2012.2213815

[160] Paramvir Bahl, Venkata N. Padmanabhan. 2005. RADAR: An In- Building RF-based User Location and Tracking System. Microsoft Research

[161] Yokoo, K.; Beauregard, S.; Schneider, M., "Indoor Relative Localization with Mobile Short-Range Radar," in Vehicular Technology Conference, 2009. VTC Spring 2009. IEEE 69th, 
vol., no., pp.1-5, 26-29 April 2009. doi: 10.1109/VETECS.2009.5073602

[162] L. Zhao, E. T. Psota and L. C. Pérez, "A comparison between UWB and TDOA systems for smart space localization," IEEE International Conference on Electro/Information Technology, Milwaukee, WI, 2014, pp. 179-183.

[163] Hazas, M., Hopper, A; A Novel Broadband Ultrasonic Location System for Improved Indoor Positioning, IEEE Transactions on mobile Computing, Vol. 5, No. 5, May 2006.

[164] A. Harter and A. Hopper,"A distributed location system for the active office", IEEE Network, vol. 8, no.1, 1994, pp. 62-70.

[165] Active Badge System, Web Site, 2008, http://www.cl.cam.ac.uk/research/dtg/attarchive/ab.html

[166] T. King, S. Kopf, T. Haenselmann, C. Lubberger and W. Effelsberg,'COMPASS: A Probabilistic Indoor Positioning System Based on 802.11 and Digital Compasses", Proc. First ACM Intl Workshop on WirelessNetwork Testbeds, Experimental evaluation and CHaracterization (WiNTECH),

[167] Hong-shik Kim; Jong-Suk Choi; Minyoung Park, "Indoor localization system using multi-modulation of ultrasonic sensors and digital compass," in Intelligent Robots and Systems, 2008. IROS 2008. IEEE/RSJ International Conference on, vol., no., pp.1359-1364, 22-26 Sept. 2008. doi: 10.1109/IROS.2008.465082.

[168] WhereNet Web Site , 2008, http://edu.symbol.com/docent authorware/WhereNet/intro/intro, last accessed on November 2010

[169] Zebra Technology Company Web Site, 2008, http://www.wherenet.com/

[170] F. Subhan, H. Hasbullah, A. Rozyyev, and S.T. Bakhsh, "Indoor positioning in Bluetooth networks using fingerprinting and lateration approach," in Proceedings of the International Conference on Information Science and Applications (ICISA '11), April 2011.

[171] H. J. Perez Iglesias, V. Barral, and C. J. Escudero, "Indoor person localization system through RSSI Bluetooth fingerprinting," in Proceedings of the 19th International Conference on Systems, Signals and Image Processing (IWSSIP '12), pp. 40-43,April 2012.

[172] Sungil Kim; Sunhwa Ha; Saad, A.; Juho Kim, "Indoor positioning system techniques and security," in e-Technologies and Networks for Development (ICeND),2015 Forth International Conference on, vol., no., pp.1-4, 21-23 Sept. 2015 